\title{
Isogeometric analysis of nonlinear Euler-Bernoulli beam vibrations
}

\author{
O. Weeger · U. Wever · B. Simeon
}

Received: date / Accepted: date

\begin{abstract}
In this paper we analyze the vibrations of nonlinear structures by means of the novel approach of isogeometric finite elements. The fundamental idea of isogeometric finite elements is to apply the same functions, namely BSplines and NURBS (Non-Uniform Rational B-Splines), for describing the geometry and for representing the numerical solution. In case of linear vibrational analysis, this approach has already been shown to possess substantial advantages over classical finite elements, and we extend it here to a nonlinear framework based on the harmonic balance principle. As application, the straight nonlinear Euler-Bernoulli beam is used, and overall, it is demonstrated that isogeometric finite elements with B-Splines in combination with the harmonic balance method are a powerful means for the analysis of nonlinear structural vibrations. In particular, the smoother $k$-method provides higher accuracy than the $p$-method for isogeometric nonlinear vibration analysis.
\end{abstract}

Keywords Isogeometric analysis - finite element method nonlinear vibration $\cdot$ harmonic balance $\cdot$ nonlinear beam

\section{Introduction}

The efficient computation of structural vibrations plays an important role in civil and mechanical engineering. Examples are freestanding structures like bridges or skyscrapers, ride comfort analysis in automobiles, and the flapping of airplane wings [1]. Today, the development of these structures

Oliver Weeger · Bernd Simeon

TU Kaiserslautern, Felix-Klein-Center for Mathematics

Paul-Ehrlich-Straße 31, 67663 Kaiserslautern, Germany

Oliver Weeger · Utz Wever

Siemens AG, Corporate Technology

Otto-Hahn-Ring 6, 81739 Munich, Germany

E-mail: oliver.weeger.ext@siemens.com relies heavily on computer-aided design (CAD), and the resulting geometry models serve as technical specification for the manufacturing process but are also the basis of static and dynamic calculations using the finite element method (FEM) [2].

Since the geometry description within the most widespread CAD programs makes use of Non-Uniform Rational B-Splines (NURBS) [3-5], it needs to be adapted for polynomial-based FEM and is approximated by meshes that usually do not represent the CAD geometries exactly. Isogeometric analysis (IGA) is aimed at bridging this gap between CAD and FEM. Introduced by Hughes et. al. [6] in 2005, IGA uses NURBS instead of polynomials both for the representation of the geometry and for constructing an approximate numerical solution in the fashion of a finite element discretization.

In this paper, we explore the benefits of IGA in the field of nonlinear vibrational analysis by combining it with the method of harmonic balance. Using a well-understood nonlinear Euler-Bernoulli beam model as benchmark problem, we demonstrate that spline-based discretizations with higher smoothness lead to an improved accuracy and are thus a promising approach. Moreover, we also give an introduction to the topic of IGA and explain the main ideas behind this new paradigm in computational engineering.

There are several advantages over traditional FEM and p-FEM [7] when using isogeometric methods. In this way, it is possible to solve the governing equations of a solid or fluid mechanics application on the exact CAD geometry. Moreover, a solution space with higher smoothness can be easily generated and leads in many cases to significantly better approximation properties. So far, isogeometric analysis has been used for a number of applications [6, 8, -11], and a mathematical theory of convergence and refinement has been established [12,17]. We refer also to the mono- 
graph by Cottrell et al. [18] for an extensive exposition of the subject.

If we take a closer look at the topic of linear modal analysis, i.e., the computation of eigenfrequencies and eigenmodes, it turns out that IGA leads also here to a better accuracy than polynomial-based FEM, Cottrell et al. [9]. Even more, the inherent instabilities that are present in the high frequencies of the so-called optic branch of the spectrum for the higher order FEM do not appear when using splines with higher smoothness instead of piecewise polynomials. These observations inspired our work, but for a nonlinear vibrational analysis, we require a different computational approach to capture the relevant dynamics of the system.

Our choice in the nonlinear case is the harmonic balance method for the study of steady-state responses of a structure subject to periodic external forces [19-24]. We introduce here two variants, the Harmonic Balance-Newton Raphson method (HBNR) [25,-29] and the Incremental Harmonic Balance method (IHB) [30,31], and test both for the nonlinear Euler-Bernoulli beam formulation of [32]. Since the vibrations of thin beams, especially low frequency suband super-harmonic resonances, have already been investigated by a number of researchers using finite element methods, we now want to apply isogeometric analysis to this problem. Due to the coupling of high and low modes in nonlinear vibration and the much better approximation of high frequencies and modes by IGA compared to FEM [9], we also expect IGA to perform well for the analysis of steadystate harmonic resonances of the nonlinear beam.

Since beams are the most simple nonlinear solid mechanics model, the advantage of preserving the exact geometry is less important in this case, and the focus lies on the benefit of using a higher continuity of the underlying spline spaces.

The paper is organized as follows: In Section 2, we give an introduction to the main concepts of IGA and apply it to the modal analysis of a linear beam model, reproducing thus the results of [9]. The method of harmonic balance for the computation of the nonlinear response dynamics of a structure is summarized in Section 3 We then combine both approaches and study the aforementioned nonlinear beam example in Section 4 In particular, the method is validated in comparison to reference results and detailed numerical convergence studies for isogeometric analysis and B-Splinebased finite elements, i.e. the $k$ - and $p$-method, are carried out. The paper finishes with a short summary and conclusions in Section 5 .

\section{Isogeometric finite elements}

In this section, we give a short overview on the main features of isogeometric finite elements and apply it to the modal analysis of a linear beam model.

\subsection{B-Splines and NURBS}

We start with the definition and properties of $B$-Splines and Non-Uniform Rational B-Splines (NURBS), the tools that are typically used for describing geometries in computeraided design and also for representing the numerical solution in isogeometric analysis [3, 4, 6, 18]. For a detailed introduction, including proofs and computer implementations, we refer to [4], while [5] also includes an historical perspective on the topic.

Starting from a knot vector $\Xi=\left\{\xi_{1}, \ldots, \xi_{r}\right\}$, which is a non-decreasing set of knots $\xi_{i} \in \mathbb{R}(i=1, \ldots, r), \xi_{i} \leq$ $\xi_{i+1}(i=1, \ldots, r-1)$, the $B$-Spline basis functions $N_{i, p}(\xi)$ of degree $p$ are defined for $i=1, \ldots, \ell, \ell:=r-p-1$, by the following recursion:

$N_{i, 0}(\xi)=\left\{\begin{array}{ll}1 & \xi_{i} \leq \xi<\xi_{i+1} \\ 0 & \text { else }\end{array}\right.$,

$N_{i, p}(\xi)=\frac{\xi-\xi_{i}}{\xi_{i+p}-\xi_{i}} N_{i, p-1}(\xi)+\frac{\xi_{i+p+1}-\xi}{\xi_{i+p+1}-\xi_{i+1}} N_{i+1, p-1}(\xi)$.

In the following, some terminology associated with the knot vectors will be useful. The half-open interval $\left[\xi_{i}, \xi_{i+1}\right)$ is called the $i$-th knot span. If the knots $\xi_{i}$ are uniformly distributed over $\Xi$, then we call the knot vector uniform, and if the first and last knot have multiplicity $p+1$, we speak of an open knot vector. Last but not least, we use the terms degree and order of a B-Spline synonymously.

There are many useful properties of B-Spline functions, and among them we point out:

- The basis functions $N_{i, p}, i=1, \ldots, \ell$ are piecewise polynomials of degree $p$.

- Compact support: $\operatorname{supp}\left(N_{i, p}\right)=\left[\xi_{i}, \xi_{i+p+1}\right), i=1, \ldots, \ell$.

- Non-negativity: $N_{i, p}(\xi) \geq 0 \forall \xi \in\left[\xi_{1}, \xi_{r}\right], i=1, \ldots, \ell$.

- Partition of unity:

For an open knot vector there is $\sum_{i=1}^{\ell} N_{i, p}(\xi) \equiv 1 \forall \xi \in\left[\xi_{1}, \xi_{r}\right]$.

- Continuity:

The basis functions $N_{i, p}$ are $p$-times continuously differentiable ( $C^{p}$-continuous) inside a knot span and at inner knots of multiplicity $k(k \leq p)$ only $C^{p-k}$.

As examples for B-Spline functions see Fig. 1 and Fig. 2

The $k$-th derivative of a basis function $N_{i, p}^{(k)}(\xi)$ is defined recursively by

$$
\begin{aligned}
N_{i, p}^{(k)}(\xi) & :=\frac{d^{k}}{d \xi^{k}} N_{i, p}(\xi)= \\
& =p\left(\frac{N_{i, p-1}^{(k-1)}(\xi)}{\xi_{i+p}-\xi_{i}}-\frac{N_{i+1, p-1}^{(k-1)}(\xi)}{\xi_{i+p+1}-\xi_{i+1}}\right) .
\end{aligned}
$$




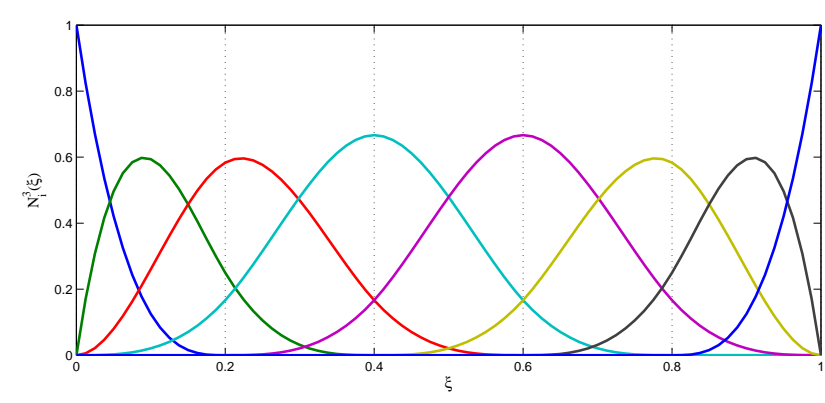

Fig. 1 B-Spline basis functions of order $p=3$ with $\ell=8$ on the open uniform knot vector $\Xi=\{0,0,0,0,0.2,0.4,0.6,0.8,1,1,1,1\}$ with inner continuity $C^{2}$

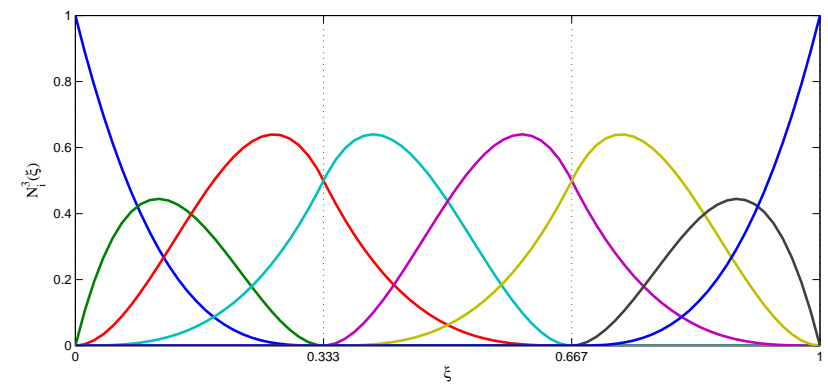

Fig. 2 B-Spline basis functions of order $p=3$ with $\ell=8$ on the open uniform knot vector $\Xi=\{0,0,0,0,0 . \overline{3}, 0 . \overline{3}, 0 . \overline{6}, 0 . \overline{6}, 1,1,1,1\}$ with inner continuity $C^{1}$

A $B$-Spline curve of order $p$ for the open knot vector $\Xi$ with control points $\mathbf{B}_{i} \in \mathbb{R}^{d}(i=1, \ldots, \ell)$ has the form

$\mathbf{C}(\xi)=\sum_{i=1}^{\ell} N_{i, p}(\xi) \mathbf{B}_{i}$.

It has the following properties:

- The polygon formed by the control points $\left\{\mathbf{B}_{i}\right\}$ is called control polygon.

- Convex hull property:

The curve is completely contained in its control polygon.

- Interpolation of start and end points:

$\mathbf{C}\left(\xi_{1}\right)=\mathbf{B}_{1}, \mathbf{C}\left(\xi_{r}\right)=\mathbf{B}_{\ell}$.

- Affine invariance:

Affine transformations of the curve can be performed on its control points.

- Displacing a control point $\mathbf{B}_{i}$ only influences the curve locally in the knot interval $\left[\xi_{i}, \xi_{i+p+1}\right)$.

- The continuity properties of the curve correspond to the ones of B-Spline functions.

In this paper, we will only use B-Spline functions and B-Spline curves for open uniform knot vectors on $[0,1]$ with inner knots of multiplicity $1 \leq k \leq p$.

In analogy to (1), the NURBS basis functions $R_{i, p}$ of order $p$ with weights $w_{i} \in \mathbb{R}(i=1, \ldots, \ell)$ for the open knot
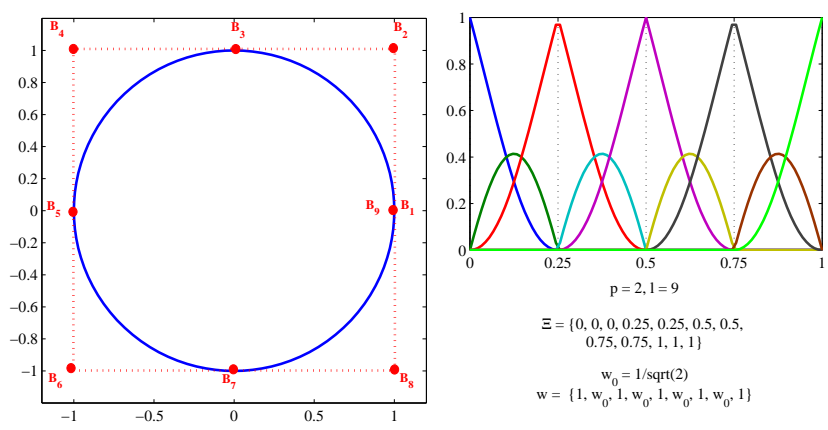

Fig. 3 A circle as a NURBS curve

vector $\Xi$ are defined by

$R_{i, p}(\xi)=\frac{N_{i, p}(\xi) w_{i}}{\sum_{j=1}^{\ell} N_{j, p}(\xi) w_{j}}$.

A NURBS curve reads, cf. (3),

$\mathbf{C}(\xi)=\sum_{i=1}^{\ell} R_{i, p}(\xi) \mathbf{B}_{i}$.

Most properties of B-Splines hold for NURBS as well. For equal weights $w_{i}=$ const $. \forall i=1, \ldots, \ell$, NURBS correspond to B-Spline basis functions. Derivatives of NURBS are more complicate than those of B-Splines and are described in detail in [4], along with efficient algorithms for their evaluation.

The main advantage of NURBS over B-Splines is the possibility to represent conic intersections exactly, which is very important for typical engineering shapes (see for example a circle in Fig. 33.

\subsection{Isogeometric analysis}

As discussed in the Introduction, the main idea behind isogeometric analysis (IGA) is to bridge the gap between CAD and FEM. Based on [6] and [18], we summarize next the major properties and discuss the relation to classical finite elements.

The key concept of IGA consists of using the NURBS curves and basis functions introduced in the previous section 2.1 both for representing the geometry and for spanning the solution spaces in the finite element method. The isoparametric concept of classical FEM employs also the same function spaces for geometry and solution representation, but there a conversion step is applied where the CAD geometry is approximated by piecewise polynomials. IGA with NURBS, on the other hand, makes it possible to bypass this conversion and mesh generation step, and, moreover, offers higher continuity of the numerical approximation.

Starting from the weak form of a partial differential equation on a domain $\Omega \subset \mathbb{R}^{d}$ with a bilinear form $a(\cdot, \cdot)$, 
linear form $l(\cdot)$ and Dirichlet boundary conditions $\left.u\right|_{\delta \Omega}=0$ we seek a solution $u$ in a corresponding function space $\mathscr{S}$ with test functions $v$ in a function space $\mathscr{V}$,

$u \in \mathscr{S}: \quad a(v, u)=l(v) \forall v \in \mathscr{V}$.

The parameterization of the domain $\Omega$ is given in terms of a geometry function on a parameter domain $\Omega_{0}$

$\mathbf{F}: \Omega_{0} \rightarrow \Omega, \mathbf{x}=\mathbf{F}(\xi)$,

that corresponds to a NURBS curve in the case of one-dimensional IGA,

$\mathbf{x}=\mathbf{F}(\xi)=\sum_{i=1}^{\ell} R_{i, p}(\xi) \mathbf{B}_{i}$

The integrals over the physical domain $\Omega$ in $a(\cdot, \cdot)$ and $l(\cdot)$ are then transformed and computed on the parameter domain $\Omega_{0}$. In the same fashion, the derivatives of $u(\mathbf{x})$ with respect to $\mathbf{x}$ can be reduced to derivatives with respect to $\xi$ using the chain rule. Therefore, it is necessary to compute the Jacobian $\mathbf{D F}(\xi)=\left(\partial F_{i} / \partial \xi_{j}\right)_{i j}$ and eventually the Hes$\operatorname{sian} \mathbf{H}_{F}(\xi)=\left(\partial^{2} F_{i} / \partial \xi_{j} \partial \xi_{k}\right)_{i j k}$.

Next, a Galerkin projection is applied, i.e., the infinitedimensional function spaces $\mathscr{S}$ and $\mathscr{V}$ are approximated by finite-dimensional subspaces $\mathscr{S}_{h} \subset \mathscr{S}$ and $\mathscr{V}_{h} \subset \mathscr{V}$. In case of IGA, the spaces spanned by the NURBS basis of the geometry function $\mathbf{F}$, eventually restricted by Dirichlet boundary conditions, are chosen for this purpose,

$$
\begin{aligned}
& \mathscr{S}_{h} \subset \operatorname{span}\left\{R_{i, p} \circ \mathbf{F}^{-1}\right\} \quad, \quad u_{h}=\sum_{i=1}^{\ell_{u}} R_{i, p}(\xi) u_{i} \\
& \mathscr{V}_{h} \subset \operatorname{span}\left\{R_{i, p} \circ \mathbf{F}^{-1}\right\} \quad, \quad v_{h}=\sum_{i=1}^{\ell_{v}} R_{i, p}(\xi) v_{i} .
\end{aligned}
$$

Substituting $u_{h}$ and $v_{h}$ from (9) into the weak form (6) yields the stiffness matrix $\mathbf{K}$ and the load vector $\mathbf{f}$,

$$
\begin{aligned}
\mathbf{K}_{i j} & =a\left(R_{i, p}, R_{j, p}\right) & & , i=1, \ldots, \ell_{v}, j=1, \ldots, \ell_{u} \\
\mathbf{f}_{i} & =l\left(R_{i, p}\right) & & , i=1, \ldots, \ell_{v} .
\end{aligned}
$$

As usual, the displacement vector $\mathbf{u}=\left\{u_{i}\right\}$ is then computed by solving a linear system

$\mathbf{K} \mathbf{u}=\mathbf{f}$,

which results in the approximate displacement $u_{h}$ in the form of a NURBS curve.

Typically, the entries of $\mathbf{K}$ and $\mathbf{f}$ are computed by quadrature rules at the element level and then assembled into the global stiffness matrix and load vector. This procedure is very similar to the classical FEM, with knot spans playing the role of elements, see [2, 15] for more details.

\subsection{Isogeometric analysis and p-FEM}

As mentioned earlier, an important advantage of isogeometric analysis with B-Splines or NURBS over classical and higher order finite elements ( $p$-FEM, [7]) is higher continuity at element boundaries. While the distinguishing mark of a finite element is its parameterization by means of a reference element, in IGA the knot spans $\left[\xi_{i}, \xi_{i+1}\right)$ for two successive knots $\xi_{i} \neq \xi_{i+1}$ are referred to as elements, and all elements of one parameter domain form a patch [18].

The typical finite element methods used for linear and nonlinear Euler-Bernoulli beams are $C^{1}$-continuous over element boundaries. In contrast to that, IGA with B-Splines and NURBS offers $C^{p-1}$-continuity at the inner knots.

To study the benefits of higher continuity and compare the isogeometric $k$ - and $p$-methods, we use B-Splines and for the $p$-method we reduce the continuity at element boundaries to $C^{1}$ with inner knots of multiplicity $p-1$. In this case, however, not the number of elements $s$ should be taken as a reference for comparing convergence properties and accuracy. Instead, the number of curve control points $\ell=\ell_{u}=\ell_{v}$ of the B-Spline curve is used since it means the same number of degrees of freedom (d.o.f.) and a similar cost of computing. Thereby we use the following rules of thumb for the number $\ell$ :

- General rule for $k$-times repeated inner knots $\left(C^{p-k}\right)$ : $\ell=p+1+k \cdot(s-1)$.

- IGA ansatz with $k=1\left(C^{p-1}\right)$ : $\ell=s+p$.

- FEM ansatz with $k=p-1\left(C^{1}\right)$ : $\ell=s \cdot(p-1)+2$.

For the total number of degrees of freedom, which are only displacement d.o.f., we have $n=d \cdot \ell$, with $d$ being the dimension of the space that the spline curve lives in $(d=1$ in the linear and $d=2$ in the nonlinear case).

Classical finite element methods provide the possibility of mesh refinement ( $h$-refinement) for increasing the accuracy, and sometimes also order elevation ( $p$-refinement, $p$ FEM). In isogeometric analysis, we have $h$-refinement (insertion of additional knots) and $p$-refinement (elevation of the order of basis functions) available as well, but there exists also a $k$-refinement procedure, which means increasing the continuity along with the order. Details on these approaches and algorithms can be found in [4,6]. For our purposes, we will only make use of knot insertion for linear parameterizations, cf. Sec. 2.4

In this paper we adopt the terminology of [9] and use the expression isogeometric analysis or IGA as synonym for parameterization and discretization with B-Splines. There is the $k$-method with simple inner knots, where we can $h$-refine for fixed degree $p$ maintaining $C^{p-1}$-continuity and degreeelevate increasing the continuity, and the $p$-method with inner knots of multiplicity $p-1$, where we can also $h$-refine 


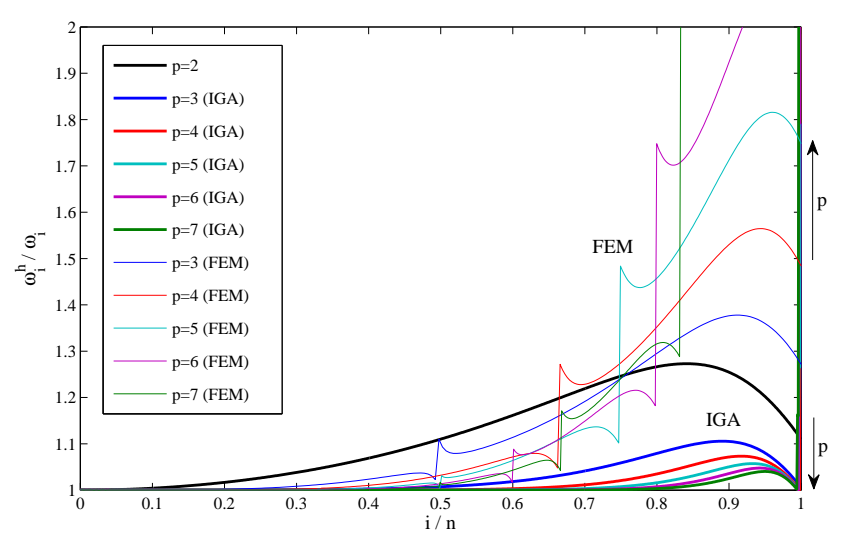

Fig. 4 Linear beam: comparison of discrete spectra for $C^{1}$-continuous $p$-method (FEM) and $C^{p-1}$-continuous $k$-method (IGA) for different degrees $2 \leq p \leq 7$

for fixed $p$ and degree elevate with constant $C^{1}$-continuity at element boundaries. We compare these methods with literature references using standard finite elements or $p$-FEM resp. higher order finite elements, which are $C^{1}$-continuous over element boundaries, just like the $p$-method.

\subsection{Isogeometric analysis of linear beam vibrations}

As a first example to show the advantages of the higher smoothness of the isogeometric method, we review next the results obtained by Cottrell et. al. [9] when computing the natural frequencies of linear Euler-Bernoulli beams. In Section 4 , we will study the isogeometric approach for nonlinear beams.

We start from the equation for the transverse displacement $w$ of a planar beam, with parameters density per unit length $\rho$, Young's modulus $E$, cross-section area $A$ and second moment of area $I$ according to [33],

$E I w^{I V}(x, t)+\rho A \ddot{w}(x, t)=p(x, t) \quad \forall x \in \Omega \times(0, T)$.

The equation for $w(x)$ can be derived by separation of variables, which leads to the following differential equation for a simply supported beam with unit parameters [9],

$$
\begin{aligned}
w^{I V}(x)-\omega^{2} w(x) & =0 \quad \forall x \in(0,1), \\
w(0)=w(1)=w^{\prime \prime}(0)=w^{\prime \prime}(1) & =0 .
\end{aligned}
$$

Here, it is possible to calculate the natural frequencies analytically [9,33] as

$\omega_{i}=(i \pi)^{2} \quad, \quad i=1,2,3, \ldots$.

In order to compute numerical values of the natural frequencies $\omega_{n}$ we derive the weak form of 13 .

$$
\int_{0}^{1} w^{\prime \prime}(x) v^{\prime \prime}(x) d x+\omega^{2} \int_{0}^{1} w(x) v(x) d x=0 .
$$

For the parameterization of the $x$-coordinate we now use a B-Spline curve of degree $p$ with open knot vector $\Xi$ and control points $\mathbf{B}_{i} \in \mathbb{R}(d=1 \Rightarrow \ell=n)$,

$x(\xi)=\mathbf{C}(\xi)=\sum_{i=1}^{\ell} N_{i, p}(\xi) \mathbf{B}_{i}$.

Following the isoparametric concept, we employ another BSpline curve for the deformation $w$ and for the test functions $v$,

$w(x)=\sum_{i=1}^{\ell} N_{i, p}(x) \mathbf{u}_{i}$.

The weak form (15) of (13) with the isogeometric discretization (16) \& 17] leads to the well-known discrete eigenvalue problem for the natural frequencies $\omega_{i}$ and natural modes $\phi_{i}$

$\left(\mathbf{K}-\omega_{i}^{2} \mathbf{M}\right) \phi_{i}=\mathbf{0} \quad, i=1, \ldots, n$

with stiffness and mass matrices

$$
\begin{aligned}
\mathbf{K}_{i j} & =\int_{0}^{1} N_{i, p}^{\prime \prime} N_{j, p}^{\prime \prime} d x, \\
\mathbf{M}_{i j} & =\int_{0}^{1} N_{i, p} N_{j, p} d x .
\end{aligned}
$$

In order to compare the numerical values of the natural frequencies $\omega^{h}$ from (18) by either isogeometric analysis with B-Splines (or NURBS) or classical polynomial-based finite elements with the exact values from (14), we compute, as in [9], normalized frequency spectra $\omega_{i}^{h} / \omega_{i}$ and plot them against $i / n$. Note that in the isogeometric case, it is even possible to derive these spectra analytically from the discretized equation of motion [9].

Figure 4 shows the comparison between the $k$ - and $p$ method (IGA resp. FEM) for $p=2, \ldots, 7$ using a linear parameterization $(d x / d \xi \equiv 1)$. While the $p$-method leads to optical and acoustical branches in the spectra for $p \geq 3$ and a very low accuracy with divergent behavior for $i / n \rightarrow 1$, the spectra for the isogeometric $k$-method are smooth with a much higher accuracy over the whole domain. Only at the end of the spectrum a few outliers occur, which are discussed in detail in [9].

However, for the $k$-method accuracy increases with finer mesh parameter $h$ and higher degree $p$, more precisely, the order of convergence is $\mathscr{O}\left(h^{2(p-1)}\right)$ [9]. While isogeometric analysis with higher order and smoothness promises a gain of accuracy, the $p$-method with $p>2$ is not suitable for analysis of higher vibration modes. Although in practice higher linear eigenfrequencies usually are not of interest, they may have an influence on the nonlinear vibrational behaviour to be studied later, due to the coupling of high and low modes. 
Since the beam formulation is rotation-free, special techniques have to be applied to enforce rotation boundary conditions like for cantilevers. Therefore we also refer to [9] where weak boundary condition imposition and the use of Lagrange multipliers are discussed in detail.

\section{Nonlinear vibration analysis with harmonic balance}

In contrast to the linear vibration case above, it is no more possible to simply set up and solve an eigenvalue problem to obtain the eigenfrequencies which describe the vibrational behavior of a structure when nonlinear models are used. Instead, the response dynamics of the structure are analyzed, and other techniques have to be applied. Our choice for determining the steady-state response of a structure subject to periodic excitation is the harmonic balance method [19. 21-24]. The application to our later example, the nonlinear Euler-Bernoulli beam, can be found in [25, 29] and [30,31].

\subsection{Nonlinear equation of motion}

The basis of vibration analysis is the dynamic equation of motion of a viscously damped structure after spatial discretization

$\mathbf{M} \ddot{\mathbf{u}}(t)+\mathbf{C} \dot{\mathbf{u}}(t)+\mathbf{K u}(t)=\mathbf{f}(t)$.

From now on, we assume a geometrical nonlinear model of the analyzed structure, which means in our case that the stiffness matrix (and eventually also the damping matrix) are depending on the deformations,

$\mathbf{K}=\mathbf{K}(\mathbf{u})$.

In order to analyze the vibrational behavior of a structure described by 20, with nonlinearity (21), we search for a periodical solution of 20 and therefore use the harmonic balance method, which will be described in detail in the following.

\subsection{Harmonic Balance-Newton Raphson method}

The main idea behind harmonic balance is that in time periodical excitation forces will lead to a periodical response behavior of the structure. That means the approximation of the solution of the equation of motion 20 can be expressed as a superposition of sine and cosine terms with different amplitudes [19,21, 24,34]. The basic procedure can be summarized as follows:

Given a periodic excitation in the frequency $\omega$ that results in a load vector

$\mathbf{f}(t)=\sum_{j=1}^{m^{*}} \mathbf{f}_{c}^{j} \cos (j \omega t)+\mathbf{f}_{s}^{j} \sin (j \omega t)$, we transform the equilibrium equation 20 to the non-dimensional time $\tau=\omega t$. With $\frac{d}{d \tau}(\cdot)=(\cdot)^{\prime}$, we obtain the differential equation system

$$
\begin{aligned}
& G\left(\mathbf{u}, \mathbf{u}^{\prime}, \mathbf{u}^{\prime \prime}, \mathbf{f}, \omega, \tau\right):= \\
& \omega^{2} \mathbf{M} \mathbf{u}^{\prime \prime}(\tau)+\omega \mathbf{C u}^{\prime}(\tau)+\mathbf{K}(\mathbf{u}) \mathbf{u}(\tau)-\mathbf{f}(\tau)=\mathbf{0} .
\end{aligned}
$$

Assuming that periodic excitation results in a periodic response from the structure, we apply a Galerkin discretization to the time domain and take a truncated Fourier series ansatz with $m$ sine and $m+1$ cosine terms (harmonics) in $\tau$ for the approximation of displacement or control point displacement degrees of freedom $\mathbf{u}$. This leads to the representation

$\mathbf{u}(\tau)=\frac{1}{2} \mathbf{a}^{0}+\sum_{j=1}^{m} \mathbf{a}^{j} \cos (j \tau)+\mathbf{b}^{j} \sin (j \tau)$.

This expression with the amplitude vector

$\mathbf{A}=\left(\mathbf{a}^{0^{T}} \mathbf{a}^{1^{T}} \ldots \mathbf{a}^{m T} \mathbf{b}^{1^{T}} \ldots \mathbf{b}^{m T}\right)^{T}$

of length $n \cdot(2 m+1)$ is substituted into 23 in order to obtain a residual

$\varepsilon(\tau)=G(\mathbf{A}, \mathbf{f}, \omega, \tau) \stackrel{!}{=} \mathbf{0}$

Now we apply the method of weighted residuals by multiplying the residual $\varepsilon(\tau)$ with the Galerkin basis functions $\cos (k \tau)$ and $\sin (k \tau)$ and integrating the equations for $\tau$ over $[0,2 \pi]$. This gives us a new system of $n \cdot(2 m+1)$ equations, which is equivalent to the first $m$ real Fourier coefficients $\mathbf{c}^{0}, \mathbf{c}^{1}, \ldots, \mathbf{c}^{m}, \mathbf{d}^{1}, \ldots, \mathbf{d}^{m}$ of the Fourier series expansion of the residual $\varepsilon(\tau)$ vanishing [21,35]:

$$
\begin{aligned}
\mathbf{c}^{k}=\int_{0}^{2 \pi} \varepsilon(\tau) \cos (k \tau) d \tau & \stackrel{!}{=} \mathbf{0} \quad, \quad k=0, \ldots, m, \\
\mathbf{d}^{k}=\int_{0}^{2 \pi} \varepsilon(\tau) \sin (k \tau) d \tau & \stackrel{!}{=} \mathbf{0} \quad, \quad k=1, \ldots, m .
\end{aligned}
$$

In order to solve this nonlinear system, i.e., to determine the coefficients $\mathbf{A}$ for known $\omega$ and $\mathbf{f}$, we use Newton's method, which is why the procedure is called Harmonic Balance-Newton Raphson Method (HBNR) [19]. The derivatives of the Fourier coefficient vectors $\mathbf{c}^{k}$ and $\mathbf{d}^{k}$ are 
found as, compare 24,

$$
\begin{gathered}
\frac{\delta c_{i}^{k}}{\delta a_{l}^{j}}=\int_{0}^{2 \pi}\left[-j^{2} \omega^{2} \mathbf{M}_{i l} \cos (j \tau)-j \omega \mathbf{C}_{i l} \sin (j \tau)\right. \\
\left.+\mathbf{K}_{T, i l} \cos (j \tau)\right] \cos (k \tau) d \tau \\
\frac{\delta c_{i}^{k}}{\delta b_{l}^{j}}=\int_{0}^{2 \pi}\left[-j^{2} \omega^{2} \mathbf{M}_{i l} \sin (j \tau)+j \omega \mathbf{C}_{i l} \cos (j \tau)\right. \\
\left.+\mathbf{K}_{T, i l} \sin (j \tau)\right] \cos (k \tau) d \tau, \sin (j \tau) \\
\frac{\delta d_{i}^{k}}{\delta a_{l}^{j}}=\int_{0}^{2 \pi}\left[-j^{2} \omega^{2} \mathbf{M}_{i l} \cos (j \tau)-j \omega \mathbf{C}_{i l}\right. \\
\left.+\mathbf{K}_{T, i l} \cos (j \tau)\right] \sin (k \tau) d \tau \\
\frac{\delta d_{i}^{k}}{\delta b_{l}^{j}}=\int_{0}^{2 \pi}\left[-j^{2} \omega^{2} \mathbf{M}_{i l} \sin (j \tau)+j \omega \mathbf{C}_{i l} \cos (j \tau)\right. \\
\left.+\mathbf{K}_{T, i l} \sin (j \tau)\right] \sin (k \tau) d \tau .
\end{gathered}
$$

Here, $\mathbf{K}_{T}$ stands for the tangential stiffness matrix, cf. Section 4.3.1 and the indices run from $k=0, \ldots, m$ resp. $k=$ $1, \ldots, m, i=1, \ldots, n, j=0, \ldots, m$ resp. $j=1, \ldots, m$ and $l=1, \ldots, n$.

The evaluation of the integrals in 27) and 28 corresponds to a Fourier transformation of the integrand $\varepsilon(\tau)$ and its derivatives, respectively, and can be done by Fast Fourier Transformation (FFT) algorithms at low computational cost and high speed.

\subsection{Incremental Harmonic Balance method}

A slightly different approach for the solution of [20], also based on the harmonic balance principle, is the Incremental Harmonic Balance method (IHB) presented with application to nonlinear beams in [30, 31].

In this variant, we also start from a periodic excitation 22 and the equation transformed to the new time $\tau$ in 23. Newton's method is then already applied in the first step. This means a linearization of the displacements $\mathbf{u}$ and frequency $\omega$ for a known state of vibration $\mathbf{u}_{0}, \omega_{0}$,

$$
\begin{aligned}
\mathbf{u} & =\mathbf{u}_{0}+\Delta \mathbf{u}, \\
\omega & =\omega_{0}+\Delta \omega .
\end{aligned}
$$

Substituting 29) into 23 and neglecting terms of second order or higher in $\Delta \mathbf{u}$ and $\Delta \omega$ results in a second order differential equation for $\Delta \mathbf{u}$ and $\Delta \omega$

$$
\begin{aligned}
& \omega_{0}^{2} \mathbf{M} \Delta \mathbf{u}^{\prime \prime}+\omega_{0} \mathbf{C} \Delta \mathbf{u}^{\prime}+ \mathbf{K}_{T}\left(\mathbf{u}_{0}\right) \Delta \mathbf{u}= \\
& \mathbf{R}-2 \omega_{0}\left(\omega_{0}^{2} \mathbf{M} \mathbf{u}_{0}^{\prime \prime}+\mathbf{C} \mathbf{u}_{0}^{\prime \prime}\right) \Delta \omega
\end{aligned}
$$

with tangential stiffness matrix $\mathbf{K}_{T}$, cf. Section 4.3.1. The residual reads

$\mathbf{R}=\mathbf{f}(\tau)-\left(\omega_{0}^{2} \mathbf{M} \mathbf{u}_{0}^{\prime \prime}+\omega_{0} \mathbf{C} \mathbf{u}_{0}^{\prime}+\mathbf{K}\left(\mathbf{u}_{0}\right) \mathbf{u}_{0}\right)$.

Following the harmonic balance principle and the Galerkin projection, we use again the truncated Fourier series ansatz with $m$ terms for the representation of $\mathbf{u}_{0}$ and $\Delta \mathbf{u}_{0}$ with respect to time $\tau[31]$,

$$
\begin{aligned}
\mathbf{u}_{0, i}(\tau) & =\frac{1}{2} \mathbf{a}_{i}^{0}+\sum_{j=1}^{m} \mathbf{a}_{i}^{j} \cos (j \tau)+\mathbf{b}_{i}^{j} \sin (j \tau) \\
& =\mathbf{C}_{S} \mathbf{A}_{i}, \\
\Delta \mathbf{u}_{0, i}(\tau) & =\frac{1}{2} \Delta \mathbf{a}_{i}^{0}+\sum_{j=1}^{m} \Delta \mathbf{a}_{i}^{j} \cos (j \tau)+\Delta \mathbf{b}_{i}^{j} \sin (j \tau) \\
& =\mathbf{C}_{S} \Delta \mathbf{A}_{i}
\end{aligned}
$$

with

$$
\begin{aligned}
& \mathbf{C}_{S}=(1 \cos (\tau) \ldots \cos (m \tau) \sin (\tau) \ldots \sin (m \tau)), \\
& \mathbf{A}_{i}=\left(\begin{array}{llllll}
\mathbf{a}_{i}^{0} & \mathbf{a}_{i}^{1} & \ldots & \mathbf{a}_{i}^{m} \mathbf{b}_{i}^{1} & \ldots & \mathbf{b}_{i}^{m}
\end{array}\right)^{T}, \\
& \Delta \mathbf{A}_{i}=\left(\Delta \mathbf{a}_{i}^{0} \Delta \mathbf{a}_{i}^{1} \ldots \Delta \mathbf{a}_{i}^{m} \Delta \mathbf{b}_{i}^{1} \ldots \Delta \mathbf{b}_{i}^{m}\right)^{T} .
\end{aligned}
$$

In matrix-vector notation we can wrap this up as

$$
\begin{aligned}
\mathbf{u}_{0}(\tau) & =\mathbf{S A}, \\
\Delta \mathbf{u}(\tau) & =\mathbf{S} \Delta \mathbf{A}
\end{aligned}
$$

with

$$
\begin{aligned}
\mathbf{S} & =\operatorname{diag}\left(\mathbf{C}_{S}, \ldots, \mathbf{C}_{S}\right), \\
\mathbf{A} & =\left(\mathbf{A}_{1}{ }^{T} \ldots \mathbf{A}_{n}{ }^{T}\right)^{T}, \\
\Delta \mathbf{A} & =\left(\Delta \mathbf{A}_{1}{ }^{T} \ldots \Delta \mathbf{A}_{n}{ }^{T}\right)^{T} .
\end{aligned}
$$

Finally, substitution of (34) into (30) and application of the method of weighted residuals, i.e. left-multiplication by $\mathbf{S}^{T}$ and integration over $[0,2 \pi]$, yields the nonlinear equation system for $\Delta \mathrm{A}$ and $\Delta \omega$

$\overline{\mathbf{K}}_{m c} \Delta \mathbf{A}=\overline{\mathbf{R}}-\overline{\mathbf{R}}_{m c} \Delta \omega$

of size $n \cdot(2 m+1)$ with

$$
\begin{aligned}
\overline{\mathbf{K}}_{m c} & =\int_{0}^{2 \pi} \omega_{0}^{2} \mathbf{S}^{T} \mathbf{M} \mathbf{S}^{\prime \prime}+\omega_{0} \mathbf{S}^{T} \mathbf{C} \mathbf{S}^{\prime}+\mathbf{S}^{T} \mathbf{K}_{T}\left(\mathbf{u}_{0}\right) \mathbf{S} d \tau, \\
\overline{\mathbf{R}} & =\int_{0}^{2 \pi} \mathbf{S}^{T} \mathbf{f}(\tau) d \tau \\
& -\int_{0}^{2 \pi} \omega_{0}^{2} \mathbf{S}^{T} \mathbf{M} \mathbf{S}^{\prime \prime}+\omega_{0} \mathbf{S}^{T} \mathbf{C} \mathbf{S}^{\prime}+\mathbf{S}^{T} \mathbf{K}\left(\mathbf{u}_{0}\right) \mathbf{S} d \tau \cdot \mathbf{A}, \\
\overline{\mathbf{R}}_{m c}= & \int_{0}^{2 \pi} 2 \omega_{0} \mathbf{S}^{T} \mathbf{M} \mathbf{S}^{\prime \prime}+\mathbf{S}^{T} \mathbf{C} \mathbf{S}^{\prime} d \tau \cdot \mathbf{A} .
\end{aligned}
$$


In order to determine a solution state $(\mathbf{A}, \omega), 36$ is solved and then $\mathbf{A}$ and $\omega$ are updated by $\Delta \mathbf{A}$ and $\Delta \omega$ as in 29. This procedure is iterated until $\Delta \mathbf{A}, \Delta \omega$ or the residual $\mathbf{R}$ of (31) is considered small enough.

In this case the integrals in (37) have to be evaluated with numerical quadrature routines that can be more costly than the FFT applied during HBNR. The advantage of IHB is its compact formulation in matrix-vector notation, although the mathematical insight into the method is more involved compared to HBNR.

Nevertheless, both methods are mathematically equivalent, as it is proven by Ferri [19].

\subsection{Response curves and continuation methods}

Analysis of nonlinear structural oscillations is usually performed by means of (frequency) response curves that show the displacement amplitudes A of (24) resp. (32) at a certain point on the structure over a certain range of the excitation frequency $\omega[20,21]$.

The simplest way to generate these response curves is to start from a fixed $\omega$ and then incrementing $\omega$ in each frequency step by a fixed or variable $\Delta \omega$ (sequential continuation, [20]). For HBNR this is equivalent to using Newton's method to obtain the state of vibration, i.e., the response amplitudes $\mathbf{A}$ for a fixed $\omega$. During the IHB procedure one has to choose $\Delta \omega=0$ in 36 and iterate until $\mathbf{R}$ and $\Delta \mathbf{A}$ are small enough. Then $\omega$ is incremented and the process starts anew.

When studying complex vibrational behavior with bifurcations, several branches and turning points, this simple method may not be sophisticated enough to follow a solution path and other techniques, so-called continuation methods have to be applied. A detailed overview and introduction to continuation methods can be found in [20]. For the implementation of HBNR we chose an arc-length method as in [26, 27], with a linear predictor $\Delta \omega$ and $\Delta \mathbf{A}$. For the IHB method we use an arc-length continuation method with cubic extrapolation as predictor for $\Delta \omega$ and $\Delta \mathbf{A}$ on the basis of [30, 36, 37].

\subsection{Further remarks on the implementation}

When using isogeometric analysis for the discretization of a structure, in our later example a beam, one has to be careful with the amplitudes A used in the truncated Fourier series expansion for the deformation degrees of freedom, 24 and (32), since these amplitudes only correspond to the control points of the B-Spline curve, not to the beam itself. In order to obtain the true amplitudes of the beam, for each harmonic $j \tau$ of (24) resp. (32) the amplitudes have to be seen as control point displacements and the displaced B-Spline curve has to be evaluated at the point of interest. For classical finite elements this is not necessary, because displacement DOFs and hence amplitudes correspond to the actual deformations. Consequently, in the following plots $a_{i}$ is not necessarily corresponding to a coefficient $a_{i}^{j}$ in $\mathbf{A}$, but to the actual amplitude of the $i$-th harmonic at the evaluation point on the beam.

Another important aspect is the convergence of the truncated Fourier series ansatz in (24) and (32), which only includes a finite number of harmonics $m$ for displacements $\mathbf{u}(\tau)$ in both cases HBNR and IHB. On the one hand no solution may be found because not enough terms are considered, on the other hand it is possible that several solutions exist for fixed $\omega$ and paths with bifurcations and turning points occur [20,21].

\section{Application of isogeometric analysis and harmonic balance to the nonlinear Euler-Bernoulli beam}

Having introduced isogeometric finite elements for structural discretization of a continuous system in Sect. 2 and harmonic balance as a means for nonlinear vibration analysis in Sect. 3. we now want to apply these two techniques to the structural model of a nonlinear Euler-Bernoulli beam [21. 32, 38, 39]. After a summary of the nonlinear beam model and its isogeometric discretization, we show some computational examples for the static case in order to verify the implementation and compare the results of the isogeometric finite elements. Then both harmonic balance methods HBNR and IHB are applied to the nonlinear vibration analysis of the beam, as in [25-31], and numerical examples will show the advantages of the isogeometric finite element $k$-method over the $p$-method.

\subsection{Nonlinear Euler-Bernoulli beam model}

A nonlinear beam model based on Euler-Bernoulli hypothesis and von Kármán strains is presented in detail in [32]. This theory, which is suitable for transverse deformations with small strains and moderate rotations, will be briefly summarized using [32] as a reference.

The displacement field $\left(u_{1}, u_{2}, u_{3}\right)$ along coordinate axis $(x, y, z)$ is expressed by the axial and transversal displacements of the neutral axis $u_{0}(x)$ and $w_{0}(x)$ (Fig. 5),

$u_{1}=u_{0}(x)-z w_{0}^{\prime}(x) \quad, \quad u_{2}=0 \quad, \quad u_{3}=w_{0}(x)$.

From the general definition of strains

$\varepsilon_{i j}=\frac{1}{2}\left(\frac{\partial u_{i}}{\partial x_{j}}+\frac{\partial u_{j}}{\partial x_{i}}\right)+\frac{1}{2} \sum_{k=1}^{3} \frac{\partial u_{k}}{\partial x_{i}} \frac{\partial u_{k}}{\partial x_{j}}$ 


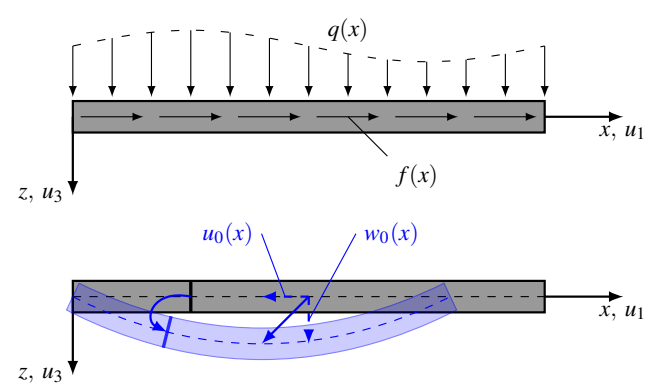

Fig. 5 Unsupported beam under external forces line load $q$ and lineedge load $f$ with transverse deformation $u_{3}$ and longitudinal deformation $u_{1}$

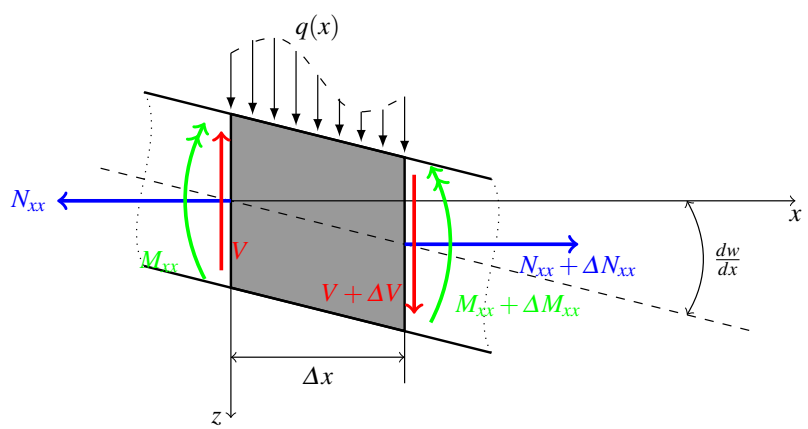

Fig. 6 Internal forces and moments on an infinitesimal beam segment

we only consider the $x x$-component of the moderately nonlinear von Kármán strain

$\varepsilon_{x x}=u_{0}^{\prime}+\frac{1}{2} w_{0}^{\prime 2}-z w_{0}^{\prime \prime}=\varepsilon_{x x}^{0}+z \varepsilon_{x x}^{1}$

with the axial strain $\varepsilon_{x x}^{0}$ and curvature $\varepsilon_{x x}^{1}$,

$\varepsilon_{x x}^{0}=u_{0}^{\prime}+\frac{1}{2} w_{0}^{\prime 2}$,

$\varepsilon_{x x}^{1}=-w_{0}^{\prime \prime}$.

From the linear constitutive law for the stress $\sigma_{x x}=E \varepsilon_{x x}$ of an isotropic beam with Young's modulus $E$, cross section area $A$ and second moment of area $I$, we obtain the internal force in $x$-direction $N_{x x}$ and the internal bending moment around $y$-axis $M_{x x}$,

$N_{x x}=E A \varepsilon_{x x}^{0}$,

$M_{x x}=E I \varepsilon_{x x}^{1}$.

The equilibrium of internal forces and moments with axial and transversal external forces $f(x)$ and $q(x)$ applied on an infinitesimal beam segment $\Delta x$ (see Fig. 6) and taking the limit $\Delta x \rightarrow 0$ leads to the governing equations

$$
\begin{aligned}
-N_{x x}^{\prime} & =f(x), \\
-\left(N_{x x} w_{0}^{\prime}\right)^{\prime}-M_{x x}^{\prime \prime} & =q(x) .
\end{aligned}
$$

Inserting equations (41) and 42) into (43) then results in the differential equations for the bending of a nonlinear
Euler-Bernoulli beam, depending on axial and transversal deformations $u_{0}$ and $w_{0}$ :

$$
\begin{array}{r}
-\left[E A\left(u_{0}^{\prime}+\frac{1}{2} w_{0}^{\prime 2}\right)\right]^{\prime}=f(x), \\
-\left[E A\left(u_{0}^{\prime}+\frac{1}{2} w_{0}^{\prime 2}\right) w_{0}^{\prime}\right]^{\prime}-\left[-E I w_{0}^{\prime \prime}\right]^{\prime \prime}=q(x) .
\end{array}
$$

\subsection{Isogeometric finite element discretization}

For a finite element discretization of the nonlinear beam formulation we need the weak forms of governing equations (43) resp. (44). According to [32] these will be derived directly from the strong form 43 using the method of weighted residuals, i.e. multiplying the equations with test functions $v_{1}$ resp. $v_{2}$, taking the integral over the length of the beam $L$ and applying integration by parts once resp. twice:

$$
\begin{aligned}
& \int_{0}^{L} N_{x x} \cdot v_{1}^{\prime} d x=\int_{0}^{L} f \cdot v_{1} d x+\left[N_{x x} \cdot v_{1}\right]_{0}^{L}, \\
& \int_{0}^{L}-M_{x x} \cdot v_{2}^{\prime \prime}+w_{0}^{\prime} \cdot N_{x x} \cdot v_{2}^{\prime} d x= \\
& \quad \int_{0}^{L} q \cdot v_{2} d x+\left[\left(M_{x x}^{\prime}+w_{0}^{\prime} \cdot N_{x x}\right) \cdot v_{2}\right]_{0}^{L}+\left[M_{x x} \cdot v_{2}^{\prime}\right]_{0}^{L} .
\end{aligned}
$$

Therefore the deformations $u_{0}$ and $w_{0}$, as well as test functions $v_{1}$ and $v_{2}$ must be included in appropriate function spaces $\mathscr{S}_{u}, \mathscr{S}_{w}, \mathscr{V}_{u}, \mathscr{V}_{w}$ and satisfy prescribed deformation boundary conditions [2].

To solve the weak equations 45, Galerkin discretization is applied, i.e., the problem is solved on a mesh $\Omega_{h}$ with finite-dimensional function spaces $\mathscr{S}_{u}^{h} \subset \mathscr{S}_{u}, \mathscr{S}_{w}^{h} \subset$ $\mathscr{S}_{w}, \mathscr{V}_{u}^{h} \subset \mathscr{V}_{u}, \mathscr{V}_{w}^{h} \subset \mathscr{V}_{w}$ defined on $\Omega_{h}$. Then $u_{0}$ and $w_{0}$ can be expressed as

$u_{0}(x)=\sum_{i=1}^{\ell_{u}} u_{i} \psi_{i}(x) \quad, \quad w_{0}(x)=\sum_{i=1}^{\ell_{w}} w_{i} \phi_{i}(x)$

with $\left\{\psi_{i}\right\}_{i=1, \ldots, \ell_{u}}$ being a basis of $\mathscr{S}_{u}^{h}$ and $\left\{\phi_{i}\right\}_{i=1, \ldots, \ell_{w}}$ a basis of $\mathscr{S}_{w}^{h}$. In the isogeometric case $\psi_{i}$ and $\phi_{i}$ are B-Spline respectively NURBS basis functions of degree $p$, and the discretized deformations are spline curves with $\ell_{u}=\ell_{w}=\ell$. Parameterization of $\mathbf{x}=\mathbf{x}(\xi)$ is as well done by means of a spline curve, cf. (16) \& (17).

Inserting these representations into (45) leads to the nonlinear system

$$
\begin{array}{ll}
\sum_{j=1}^{\ell} \mathbf{K}_{i j}^{11} u_{j} & +\sum_{J=1}^{\ell} \mathbf{K}_{i J}^{12}\left(w_{0}\right) w_{J}=\mathbf{f}_{i}^{1} \quad, i=1, \ldots, \ell, \\
\sum_{j=1}^{\ell} \mathbf{K}_{I j}^{21}\left(w_{0}\right) u_{j} & +\sum_{J=1}^{\ell} \mathbf{K}_{I J}^{22}\left(w_{0}\right) w_{J}=\mathbf{f}_{I}^{2} \quad, I=1, \ldots, \ell
\end{array}
$$


with stiffness matrices and force vectors

$$
\begin{aligned}
\mathbf{K}_{i j}^{11} & =\int_{0}^{L} E A N_{i, p}^{\prime}(x) N_{j, p}^{\prime}(x) d x, \\
\mathbf{K}_{i J}^{12}\left(w_{0}\right) & =\frac{1}{2} \int_{0}^{L} E A w_{0}^{\prime} N_{i, p}^{\prime}(x) N_{J, p}^{\prime}(x) d x, \\
\mathbf{K}_{I j}^{21}\left(w_{0}\right) & =\int_{0}^{L} E A w_{0}^{\prime} N_{I, p}^{\prime}(x) N_{j, p}^{\prime}(x) d x=2 \mathbf{K}_{j I}^{12}, \\
\mathbf{K}_{I J}^{22}\left(w_{0}\right) & =\int_{0}^{L} E I N_{I, p}^{\prime \prime}(x) N_{J, p}^{\prime \prime}(x) d x \\
& +\frac{1}{2} \int_{0}^{L} E A w_{0}^{\prime \prime} N_{I, p}^{\prime \prime}(x) N_{J, p}^{\prime \prime}(x) d x, \\
\mathbf{f}_{i}^{1} & =\int_{0}^{L} f(x) N_{i, p}(x) d x+\hat{Q}_{i}, \\
\mathbf{f}_{I}^{2} & =\int_{0}^{L} q(x) N_{I, p}(x) d x+\bar{Q}_{I}
\end{aligned}
$$

and $\hat{Q}_{i}, \bar{Q}_{I}$ being boundary terms derived from 45 . More details can be found in [32]. Next, (48) can be condensed to the following nonlinear equation system for control point deformations $\mathbf{u}$, with deformation-dependent stiffness ma$\operatorname{trix} \mathbf{K}=\mathbf{K}(\mathbf{u})$ and force vector $\mathbf{f}$ :

$\mathbf{u}=\left(\begin{array}{c}u_{i} \\ w_{I}\end{array}\right), \mathbf{K}(\mathbf{u})=\left(\begin{array}{ll}\mathbf{K}_{i j}^{11} & \mathbf{K}_{i J}^{12} \\ \mathbf{K}_{I j}^{21} & \mathbf{K}_{I J}^{22}\end{array}\right), \mathbf{f}=\left(\begin{array}{c}\mathbf{f}_{i}^{1} \\ \mathbf{f}_{I}^{2}\end{array}\right)$,

$\mathbf{K}(\mathbf{u}) \mathbf{u}=\mathbf{f}$.

\subsection{Static displacement of the nonlinear beam}

Having derived the nonlinear Euler-Bernoulli beam model with the resulting nonlinear stiffness matrix in the previous section, we now want to solve 50 to compute the displacement of the beam subject to a static load. Therefore we use Newton's method, which is described in the following, and then some computational examples are shown.

\subsubsection{Solving the nonlinear system}

For solving the nonlinear equation system w0 use a Newton-Raphson method [18, 32]. The root $\mathbf{u}^{*}$ of $\mathbf{K}(\mathbf{u}) \mathbf{u}-$ $\mathbf{f}=\mathbf{0}$ is approximated by a sequence $\mathbf{u}^{s}$ such that $\mathbf{u}^{s} \rightarrow \mathbf{u}^{*}$ :

- Starting value: $\mathbf{u}^{0}$
- Iteration step $s>0$ :

- Residual: $\quad \mathbf{r}^{s-1}=\mathbf{K}\left(\mathbf{u}^{s-1}\right) \cdot \mathbf{u}^{s-1}-\mathbf{f}$

- Corrector $\Delta \mathbf{u}^{s}$ from:

$$
\mathbf{K}_{T}^{s-1} \cdot \Delta \mathbf{u}^{s} \equiv \frac{d \mathbf{r}^{s-1}}{d \mathbf{u}^{s-1}} \cdot \Delta \mathbf{u}^{s}=-\mathbf{r}^{s-1}
$$

- Update: $\mathbf{u}^{s}=\mathbf{u}^{s-1}+\Delta \mathbf{u}^{s}$

- Convergence criteria:

$\left\|\Delta \mathbf{u}^{s}\right\|<\varepsilon \wedge\left\|\mathbf{r}^{s-1}\right\|<\varepsilon$.

According to [32], the tangential stiffness matrix $\mathbf{K}_{T}$ can be calculated explicitly and is symmetric, independent of the symmetry of $\mathbf{K}$. The entries of

$$
\mathbf{K}_{T} \equiv \frac{d \mathbf{r}}{d \mathbf{u}}=\left(\begin{array}{ll}
\mathbf{K}_{T, i j}^{11} & \mathbf{K}_{T, i J}^{12} \\
\mathbf{K}_{T, I j}^{21} & \mathbf{K}_{T, I J}^{22}
\end{array}\right)
$$

are

$\mathbf{K}_{T, i j}^{11}=\mathbf{K}_{i j}^{11}$,

$\mathbf{K}_{T, i J}^{12}=2 \mathbf{K}_{i J}^{12}$,

$\mathbf{K}_{T, I j}^{21}=\mathbf{K}_{I j}^{21}$,

$\mathbf{K}_{T, I J}^{22}=\mathbf{K}_{I J}^{22}+\int_{0}^{L} E A\left(u_{0}^{\prime}+w_{0}^{\prime 2}\right) N_{I, p}^{\prime \prime}(x) N_{J, p}^{\prime \prime}(x) d x$.

\subsubsection{Computational results for static examples}

In order to validate the isogeometric analysis of static beam deformations and study the accuracy and convergence of the method we compare the results for different support types with the ones given in [32]. There one can find the maximum deflection of the center line of the beam subject to a constant distributed load $q_{0}$. The parameters of the beam used are

$$
\begin{aligned}
& L=100 \text { in. }, \quad b=1 \text { in. }, \quad h=1 \text { in. } \\
& E=30 \mathrm{msi}, \quad I=b h^{3} / 12=1 / 12 \text { in. }^{4} .
\end{aligned}
$$

The three different types of supports of the beam with the corresponding boundary conditions applied to 45 and (48) are (see also Fig. 7):

- hinged:

$$
\begin{aligned}
& u_{0}(0)=0, \\
& w_{0}(0)=w_{0}(L)=0 .
\end{aligned}
$$

- pinned:

$$
\begin{gathered}
u_{0}(0)=u_{0}(L)=0, \\
w_{0}(0)=w_{0}(L)=0 .
\end{gathered}
$$

- clamped:

$$
\begin{aligned}
& u_{0}(0)=u_{0}(L)=0, \\
& w_{0}(0)=w_{0}(L)=w_{0}^{\prime}(0)=w_{0}^{\prime}(L)=0 .
\end{aligned}
$$




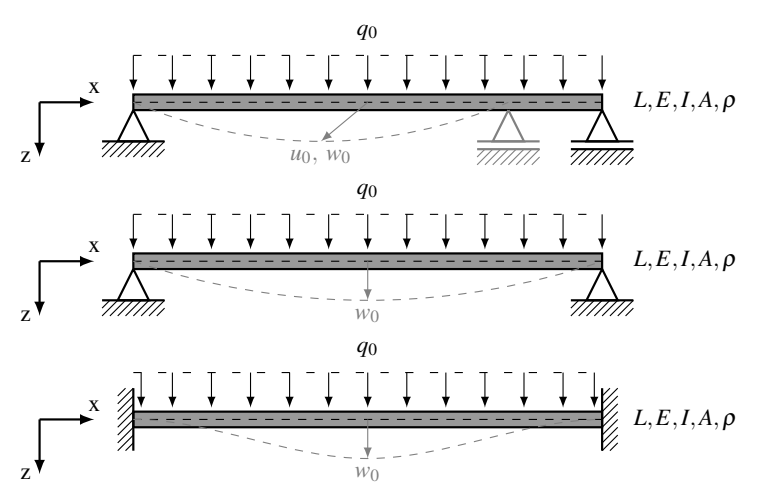

Fig. 7 hinged, pinned and clamped beam under line load $q_{0}$

For the hinged configuration the deformation in $z$-direction corresponds to the linear case, and it is possible to derive $w_{0}$ analytically [32],

$w_{0}(x)=\frac{q_{0} L^{4}}{24 E I}\left(\frac{x}{L}-2 \frac{x^{3}}{L^{3}}+\frac{x^{4}}{L^{4}}\right)$.

It follows that the maximum deformation is at the midspan of the beam $x=L / 2$ where $w_{0}(L / 2)=0.52083333$ for $q_{0}=$ 1.0 .

The classical finite element method with cubic Hermite polynomials for $w_{0}$ and linear $u_{0}$ used in [32] shows membrane locking phenomena that can only be resolved by reduced integration of the nonlinear parts of the stiffness matrix. Our results obtained with the $k$ - and $p$-method derived from the isogeometric formulation do not show these problems.

A detailed convergence study for different mesh size $h=$ $1 /(\ell-1)$ and polynomial resp. spline degree $p$, comparing the absolute error of maximum deformation $\mid w_{0}^{h}(L / 2)-$ $w_{0}(L / 2) \mid$ in Fig. 8, shows that the $k$-method (IGA) is more accurate than the $p$-method (FEM) and that the order of convergence for both cases can be estimated as $\mathscr{O}\left(h^{2(p-1)}\right)$. This corresponds to the order achieved for linear eigenfrequency computations in Sec. 2.4

For pinned (not displayed) and clamped (Fig. 9 exact reference solution $w_{0}(L / 2)=0.10335910$ in.) beam configurations the results are basically the same as for the hinged case: the isogeometric $k$-method turns out to provide higher accuracy than the $p$-method.

We can conclude for the static computations that $C^{p-1}$ continuous isogeometric analysis of nonlinear beams provides more accurate results than classical $C^{1}$-continuous finite elements, resp. the B-Spline-based $p$-method, for the same number of degrees of freedom at the same rate of convergence. So we have validated the model and can proceed to nonlinear vibration analysis using this nonlinear EulerBernoulli beam formulation and its isogeometric discretization.

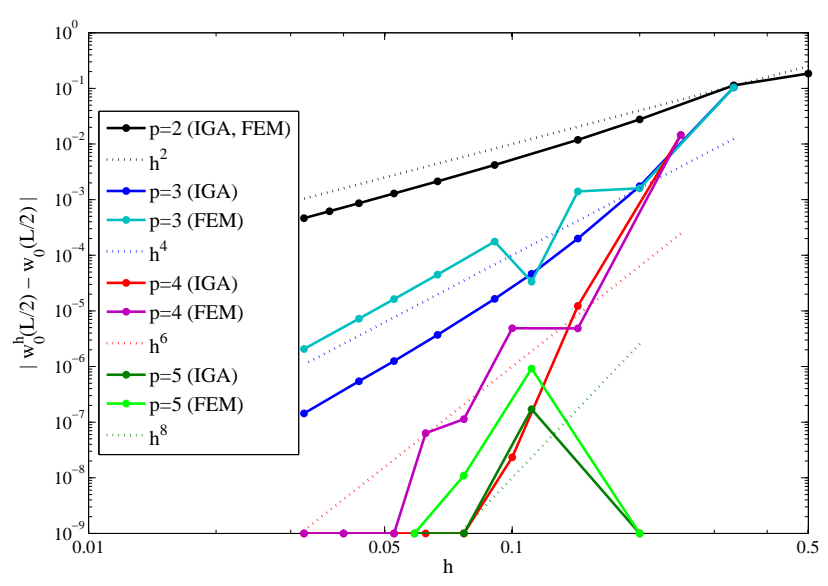

Fig. 8 Convergence plot for hinged beam: Comparison of absolute errors of $C^{1}$-continuous $p$-method (FEM) and $C^{p-1}$-continuous $k$ method (IGA) (for visualization purposes an error up to computing precision is displayed as $10^{-9}$ )

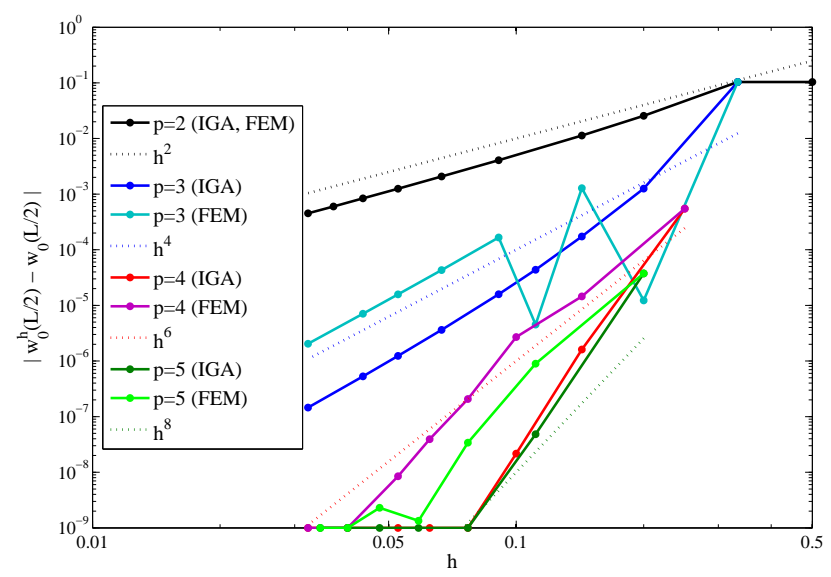

Fig. 9 Convergence plot for clamped beam: Comparison of absolute errors of $C^{1}$-continuous $p$-method (FEM) and $C^{p-1}$-continuous $k$-method (IGA) (for visualization purposes an error up to computing precision is displayed as $10^{-9}$ )

\subsection{Nonlinear beam vibrations}

For the study of nonlinear beam vibrations with isogeometric discretization using the two harmonic balance techniques HBNR and IHB as discussed in Sec. 3, we are now showing three computational examples, especially focusing on sub- and super-harmonic resonances. The first one is from Ribeiro and Petyt [27] resp. Ribeiro [28], the second one was studied before by Chen et al. [31], while the third one is a detailed convergence study comparing IGA and $p$-FEM.

The nonlinear stiffness matrix $\mathbf{K}=\mathbf{K}(\mathbf{u})$ involved in the equation of motion 20, which is the basis of vibration analysis, is given for the nonlinear Euler-Bernoulli beam in (48). As mass matrix $\mathbf{M}$ we chose the consistent one derived from the variational formulation, since the usage of lumped mass matrices showed poor results for linear eigenfrequency computations with higher order isogeometric analysis in [9]. Its 
entries are larger or equal to zero due to the non-negativity of basis functions and, denoting the mass density by $\rho$, the mass matrix reads:

$$
\begin{aligned}
& \mathbf{M}=\left(\begin{array}{ll}
\mathbf{M}_{u} & 0 \\
0 & \mathbf{M}_{w}
\end{array}\right), \\
& \mathbf{M}_{u, i j}=\rho A \int_{0}^{L} N_{i, p}(x) N_{j, p}(x) d x \quad, i, j=1, \ldots, \ell, \\
& \mathbf{M}_{w, I J}=\rho A \int_{0}^{L} N_{I, p}(x) N_{J, p}(x) d x \quad, I, J=1, \ldots, \ell .
\end{aligned}
$$

The tangential stiffness matrix $\mathbf{K}_{T}$ needed for HBNR and IHB is given in 51).

\subsubsection{Linear eigenfrequencies and internal resonance of the beam}

To validate the isogeometric approach to the nonlinear vibration analysis we start with the comparison of our results with the works of Ribeiro and Petyt [27] and Ribeiro [28], where a $p$-FEM approach, so-called hierarchical finite elements (HFEM), is used for discretization.

A beam with the following parameters is investigated in the following:

$$
\begin{gathered}
L=0.580 m, \quad h=0.002 m, \quad b=0.020 m, \\
E=7 \cdot 10^{10} \mathrm{~N} / \mathrm{m}^{2}, \quad I=1.333 \cdot 10^{-11} \mathrm{~m}^{4}, \\
r=5.774 \cdot 10^{-4} \mathrm{~m}, \quad \rho=2778 \mathrm{~kg} / \mathrm{m}^{3} .
\end{gathered}
$$

Before studying the steady-state vibration of the nonlinear beam, we want to take up the results from [27] and [28] for the linear modal analysis of the simply supported and clamped beam (see also Section 2.4 about linear beam vibrations).

For simple support boundary conditions, in both [27] and [28] the linear frequency parameter $\lambda=\omega_{1}^{2} L^{4} m / E I$ is computed using different methods and compared to the exact solution $\lambda=\pi^{4}$. The number of degrees of freedom needed to approximate $\lambda \doteq 97.409$ with 5 digits are shown in Table 1 for the classical finite element method (FEM), the HFEM method applied in [27, 28] and the exact beam eigenfunctions in [28]. In Table 2 the number of d.o.f. needed using our B-Spline approach with $C^{p-1}$-continuity ( $k$-method) and $C^{1}$-continuity ( $p$-method) are displayed for comparison. It is clear that isogeometric B-Splines cannot keep up with the use of beam eigenfunctions, but compared to the HFEM polynomial method, where only one $p$-element is employed, and standard FEM the results are satisfactory, since for $p \geq 6$ one element only is sufficient to achieve the desired accuracy as well. Again the benefit of the higher smoothness of the $k$-method compared to the $p$-method is clearly visible when more than one element has to be used for $p \leq 5$.

\begin{tabular}{|c|rrr|}
\hline Method & FEM & HFEM & eigenfunctions \\
\hline d.o.f. & 34 & 6 & 1 \\
\hline
\end{tabular}

Table 1 Number of degrees of freedom needed to approximate the exact linear frequency parameter $\lambda \doteq 97.409$ with the methods from 27. 28

\begin{tabular}{|c|rrrrr|}
\hline$p$ & 3 & 4 & 5 & 6 & 7 \\
\hline$k$-method & 15 & 7 & 6 & 5 & 6 \\
$p$-method & 28 & 12 & 8 & 5 & 6 \\
\hline
\end{tabular}

Table 2 Number of degrees of freedom needed to approximate the exact linear frequency parameter $\lambda \doteq 97.409$ with the B-Spline $k$-method and $p$-method

\begin{tabular}{|c|rrrrr|}
\hline$p$ & 3 & 4 & 5 & 6 & 7 \\
\hline$k$-method & 17 & 10 & 9 & 9 & 8 \\
$p$-method & 30 & 19 & 18 & 13 & 10 \\
\hline
\end{tabular}

Table 3 Number of degrees of freedom needed to approximate the first four eigenfrequencies of the clamped beam by B-Spline $k$-method and $p$-method

For the clamped beam it is stated in [27] that the first four eigenfrequencies $\omega_{1}, \ldots, \omega_{4}$ can be approximated on 4 digits already by the use of 5 HFEM basis functions, i.e. 5 d.o.f., taking into account the symmetries. The number of B-Spline basis functions needed can be seen in Table 3 We have a minimum of 8 for B-Splines with $p=7$ and again the $k$-method proves to be advantageous compared to the $p$ method.

Now we continue with a comparison of the results for nonlinear vibration analysis, where Ribeiro and Petyt [27] use HFEM in combination with harmonic balance and arclength continuation to determine periodic steady-state responses of a clamped beam.

Hysteretic, mass proportional damping is used with $\mathbf{C}=$ $0.01 \omega_{1}^{2} \mathbf{M}$ and the beam is excited by a harmonic point force at $x=0.25 \mathrm{~L}$. At this point the vibration amplitudes $a_{1}, a_{3}$ of the first resp. third harmonic are computed and the response curves are plotted. Due to the asymmetric excitation a 1:3 internal resonance will occur.

In Figures 10 and 11 we have generated the response curves of the first and third harmonic $a_{1}$ and $a_{3}$ near the first eigenfrequency $\omega_{1}$, using the isogeometric B-Spline method ( $p=5, s=11, \ell=16)$ with HBNR $(m=3)$ and arc-length continuation. Typical harmonic resonance behavior can be observed and the results correspond very well to the ones using HFEM with one $p$-element in [27].

The response curves around the second eigenfrequency $\omega_{2} \approx 2.76 \cdot \omega_{1}$ are shown in Figures 12 and 13 As it is pointed out in [27], here the 1:3 internal resonance occurs, 


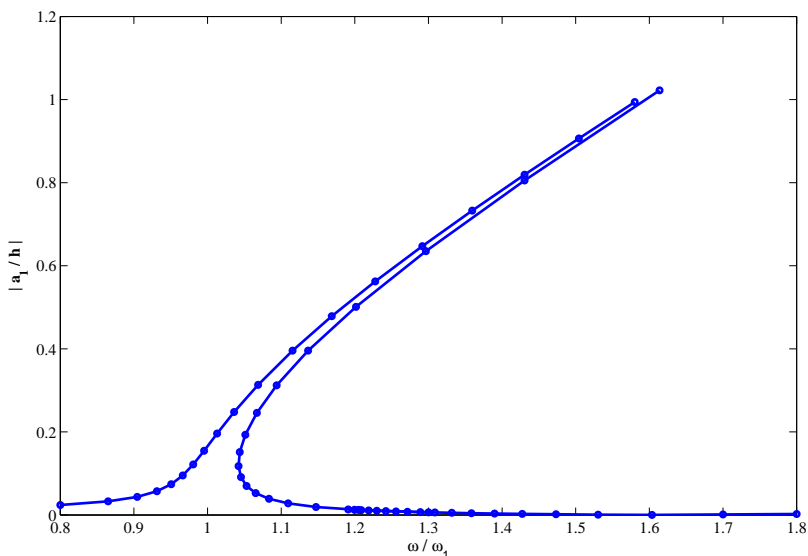

Fig. 10 Response curve of first harmonic around $\omega_{1}$, generated with HBNR with $p=5, s=11, \ell=16$

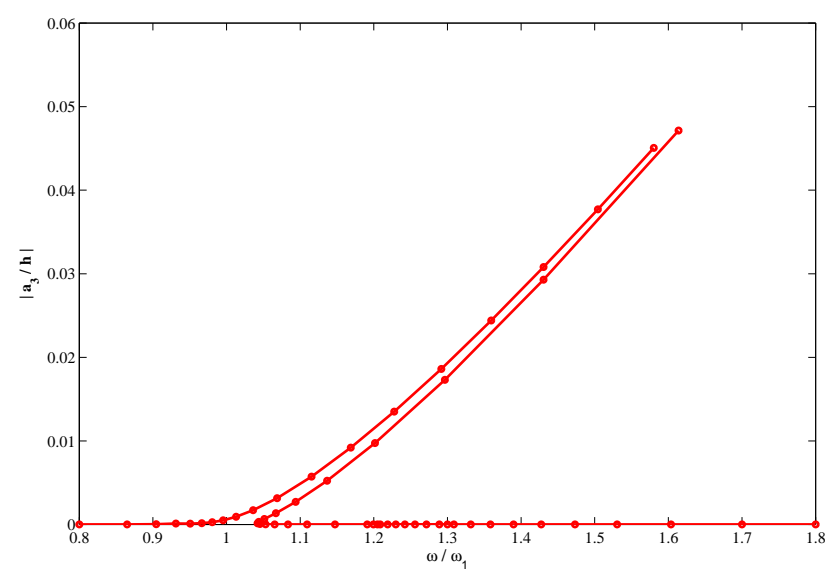

Fig. 11 Response curve of third harmonic around $\omega_{1}$, generated with HBNR with $p=5, s=11, \ell=16$

because the fourth mode gets excited, too, since $\omega_{4} \approx 3.24$. $\omega_{2}$. This leads to a different behavior for increasing amplitude and shape of the response function compared to the pure harmonic resonance. Although the shapes of the frequency response curves generated by our HBNR method are a bit different from the ones in [27] at the point where the $1: 3$ internal resonance occurs, the general correspondence is again very well.

Thus we have shown in a first example the correspondence of the qualitative results for the application of isogeometric finite elements compared to hierarchical finite elements within nonlinear vibration analysis.

\subsubsection{Combined resonance of a simply supported beam}

In order to perform another comparison with a literature example and also validate the IHB method, we want to study an example from Chen et al. [31]. There the discretization is done by a classical finite element method with polynomial degree $p_{w}=3$ in $z$-resp. $p_{u}=1$ in $x$-direction, which is $C^{1}$ at

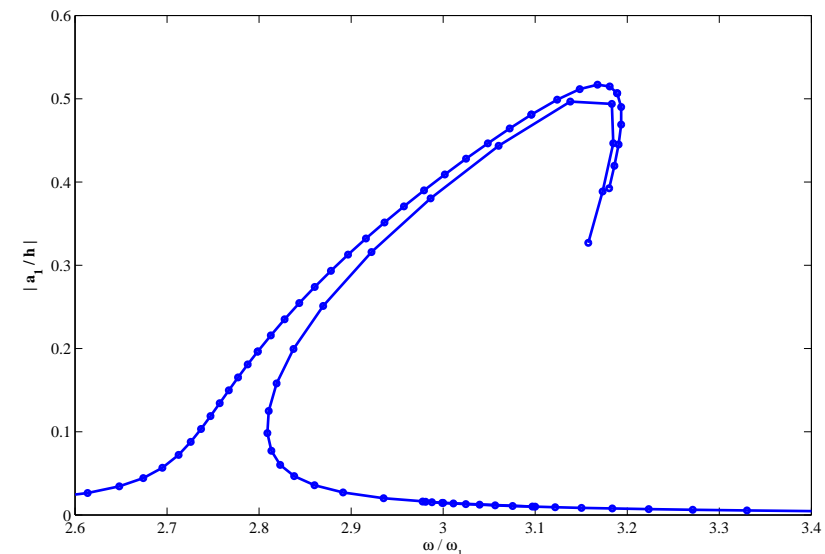

Fig. 12 Response curve of first harmonic around $\omega_{2} \approx 2.76 \cdot \omega_{1}$, generated with HBNR with $p=5, s=11, \ell=16$

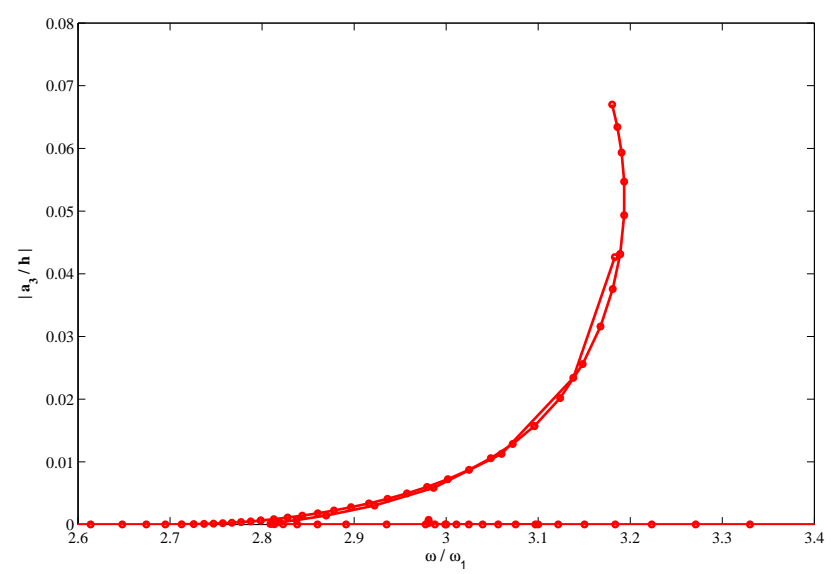

Fig. 13 Response curve of third harmonic around $\omega_{2} \approx 2.76 \cdot \omega_{1}$, generated with HBNR with $p=5, s=11, \ell=16$

element boundaries, and the incremental harmonic balance and arc-length continuation are applied to study the forced vibration of a simply supported beam with the following parameters:

$$
\begin{array}{cc}
L=1.0, \quad A=0.1, \quad E=2 \cdot 10^{5}, \quad I=0.00081 \\
r=0.09, \quad \rho=2.0, \quad \mu=0.0
\end{array}
$$

Excitation is induced by three periodic point loads, Fig. 14

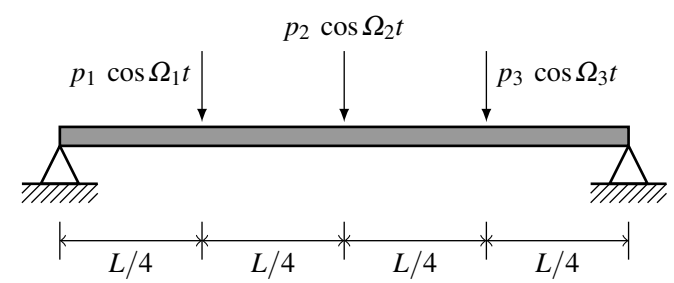

Fig. 14 Simply supported beam subject to three periodic point loads 


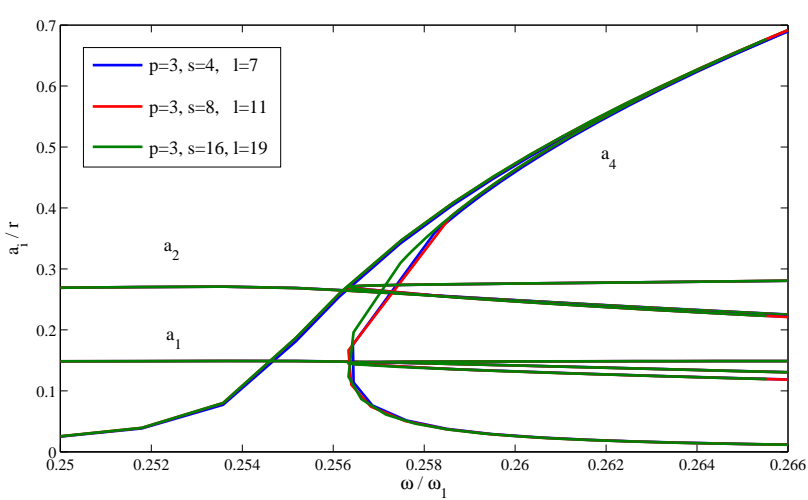

Fig. 15 Response curves B-Spline degree $p=3$ and $s=4,8,16$ elements via IHB with $m=4$ harmonic terms

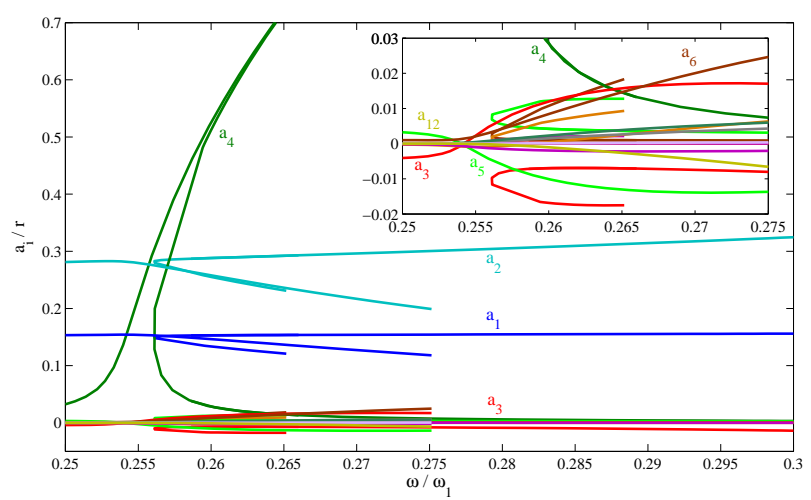

Fig. 16 Response curves B-Spline degree $p=5$ and $s=8$ elements via IHB with $m=12$ harmonic terms. Special focus on divergent higher harmonic amplitudes

with amplitudes

$p_{1}=0.5 p_{2}=p_{3}=5 \mathrm{EIr} / L^{3}$

and frequencies

$\omega=\Omega_{1}=0.5 \Omega_{2}=\Omega_{3}$.

In [31] the range around $\omega \approx \omega_{1} / 4$ is examined. There the amplitudes $a_{1}$ and $a_{2}$ corresponding to the excitation frequencies become only slightly smaller with increasing excitation frequency, while the super-harmonic term $a_{4}$ shows resonance with fast growing amplitude at $\omega / \omega_{1} \approx 0.255$. According to [31] all other coefficients (not shown) remain constantly around 0 . After passing a maximum at $\omega / \omega_{1} \approx$ 0.265 , there is a turning point in the curves of $a_{1}, a_{2}$ and $a_{4}$ and they reach their original levels again.

The results obtained by means of isogeometric analysis in this paper basically correspond to the ones shown in [31]: In Fig. 15. we have used Incremental Harmonic Balance with a B-Spline degree of $p=3, s=4,8,16$ elements. One can see that the behavior of the absolute amplitudes matches the one presented in [31] very well and that resonance in the fourth harmonic term occurs around $\omega / \omega_{1} \approx 0.255$. Even

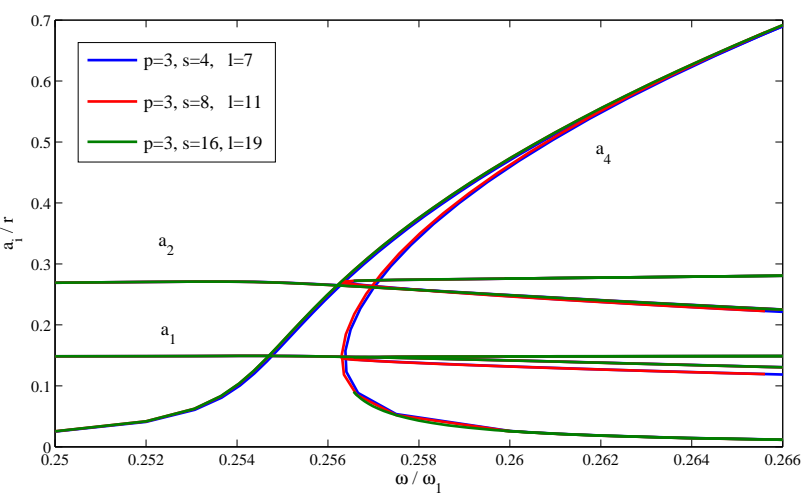

Fig. 17 Response curves B-Spline degree $p=3$ and $s=4,8$, 16 elements via HBNR with $m=4$ harmonic terms

for the low B-Spline degree and coarse discretizations the response curves are hardly distinguishable.

The results presented so far were all obtained using $m=$ 4 harmonic terms for representation of displacement d.o.f., just as in [31], but as mentioned already before in Sec. 3.5 the convergence of the truncated Fourier series ansatz has to be checked as well in order to judge results. Therefore in Fig. 16 we have used $p=5, s=8$ and $m=12$ and put special focus on the amplitudes of $a_{3}$ and $a_{k}, 5 \leq k \leq 12$ in the range of resonance in $a_{3}$. These coefficients have rising amplitudes as well, which is not in agreement with [31] where these amplitudes were considered as neglectable. So our conclusion is that it is hard to judge the response curves in this case.

The Harmonic Balance-Newton Raphson method was tested as well. In Fig. 17] we have again the response curves for the middle of the beam, now using HBNR with $p=$ $3, s=4,8,16, m=4$. Basically this plot looks identically to the one obtained with IHB, cf. Fig. 15 .

For this example we can conclude that isogeometric finite elements with both HBNR and IHB qualitatively match the results from [31] using classical FEM very well and both methods implemented provide identical results. A detailed convergence study of the $k$ - and $p$-method and comparison between the HBNR and IHB methods will be done with the next example.

\subsubsection{Convergence studies for the $k$ - and p-method}

In the previous examples the isogeometric approach to nonlinear vibration analysis with B-Splines of degree $p$ with $C^{p-1}$-continuity ( $k$-method) and $C^{1}$-continuity ( $p$-method) were validated and compared to reference work using the hierarchical resp. classical finite element discretization of the nonlinear Euler-Bernoulli beam. Now we carry out a more detailed convergence study comparing the isogeometric $k$ method with the $p$-method in order to explore the benefits of higher continuity. 


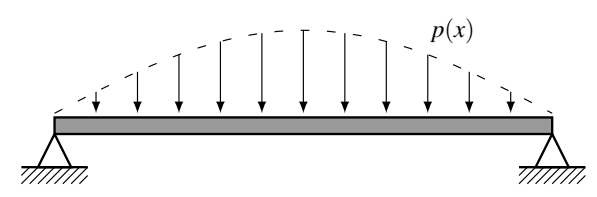

Fig. 18 Simply supported beam subject to periodic sinusoidal load

The parameters of the simply supported beam are chosen to be the same ones as in the previous example 60). As load we have a sinusoidal distributed load which is periodic in time, see Fig. 18 .

$$
\begin{aligned}
p(x, \tau)=20 E I r / L^{3} \sin (\pi x / L) \cos (\tau), & \\
x & \in[0, L], \tau \in[0,2 \pi] .
\end{aligned}
$$

For the error calculation and convergence study we focus on the mid span of the beam, where sub-harmonic resonance in $a_{3}$ occurs around $\omega / \omega_{1} \approx 0.34$. Convergence of the truncated Fourier series expansions for deformations will be studied as well, but for the beginning we will start with $m=3$ for convergence analysis with respect to $p$ and $h=$ $1 /(\ell-1)$ since the contribution of higher-frequency amplitudes above $a_{3}$ is very small.

In Fig. 19 and Fig. 20 we have the response curves for HBNR and IHB with $p=3$ and $\ell=8,12$ around $\omega / \omega_{1} \approx$ 0.34 , and one can see the resonance in $a_{3}$. Only for the $p$ method with $\ell=8$, a difference to the other curves is visible, for the other cases the curves match optically.

For the study of $p$ - and $h$-convergence of the isogeometric methods using both HBNR and IHB we have the convergence plots in Fig. 21 and Fig. 22, showing the error in the amplitude of the third harmonic $\left|a_{3}^{h}-a_{3}\right|$ at $\omega / \omega_{1}=0.338$ for $p=3,4,5$. As reference solution $a_{3}$ we take the numerical one obtained from $p=5$ and a sufficient number of DOF with an accuracy of 8 digits (error $10^{-9}$ equal to exact solution). For HBNR we have $a_{3}=0.20568197$ and for IHB $a_{3}=0.20555643$, so both methods do not exactly converge to the same value. Nevertheless we can see convergence for both methods. The order of convergence is again $\mathscr{O}\left(h^{2(p-1)}\right)$, at least for $p=3$, but in general the error for the $C^{p-1} k$-method is much smaller than for $C^{1}$-continuous $p$-method. This matches exactly the results we have already observed for linear modal analysis in Sec. 2.4 and the static deformation computation of the nonlinear beam in Sec. 4.3 .

So far we have only analyzed IHB and HBNR regarding $p$ - and $h$-convergence with the result that both methods converge with rate $\mathscr{O}\left(h^{2(p-1)}\right)$, but towards different values. One reason for this might be the influence of the number of terms $m$ used for the expression of deformations as a truncated Fourier series. So now we take a deeper look at the $m$-convergence.

In Tab. 4 we have the amplitudes $a_{3}$ at $\omega / \omega_{1}=0.336$ for different number of terms $m$ with $p=5, \ell=18$. Here,

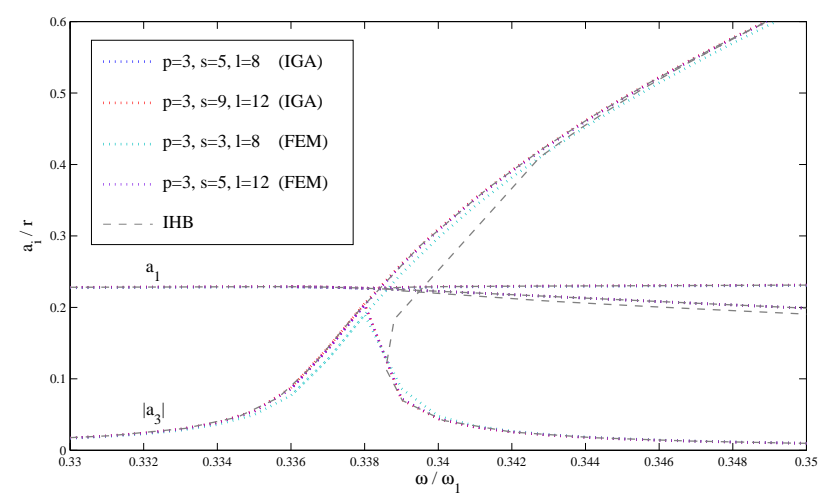

Fig. 19 Comparison of response curves for $C^{2}$ - (IGA) and $C^{1}$ continuous (FEM) cubic B-Splines using HBNR

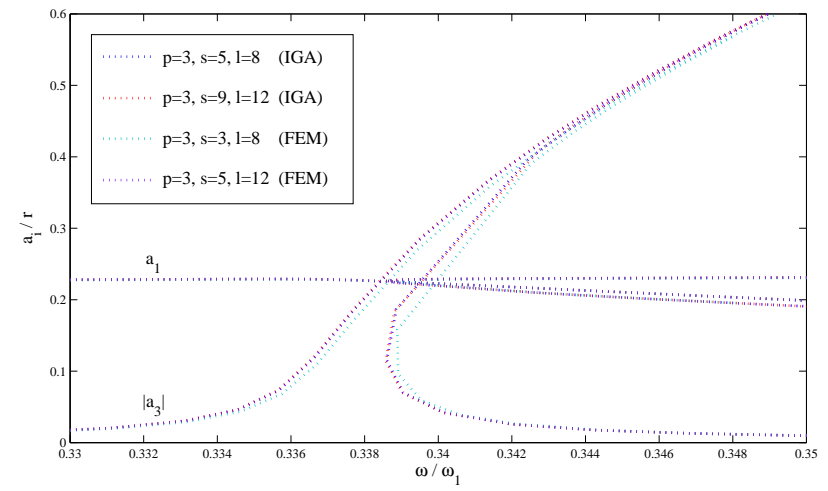

Fig. 20 Comparison of response curves for $C^{2}$ (IGA) and $C^{1}$ continuous (FEM) cubic B-Splines using IHB

$\varepsilon$ is the error in $a_{3}$ compared to the value for $m \geq 10 . \Delta$ is the difference between IHB and HBNR with an accuracy of $10^{-8}$. The $m$-convergence of both methods is clearly visible, i.e. the error is decreasing with increasing $m$ and is 0 for $m \geq 10$. For IHB and HBNR the error is of the same order of magnitude for a constant $m$, even nearly equal. However, also the difference of both methods stays practically the same; independent of $m$ the value of $\Delta$ is of magnitude $10^{-5}$.

The reason for IHB and HBNR having the same convergence behavior in $p, h$ and $m$, but converging towards slightly different values, is not clear yet. However, the use of $m=3$ for the analysis of $p$ - and $h$-convergence is justified, since the number of terms $m$ does not affect the convergence behavior in $p$ and $h$.

Furthermore, due to the cosinusoidal temporal excitation used in these examples the sinusoidal terms with the amplitudes $\mathbf{b}^{j}$ in (24) resp. (32) can be neglected without influencing the results. Thereby the total number of harmonic coefficients used in both harmonic balance methods can be reduced from $2 m+1$ to $m+1$. 


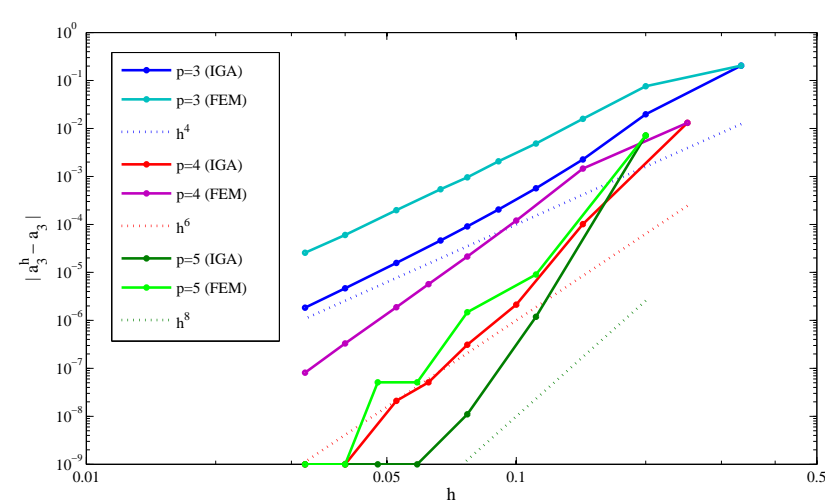

Fig. 21 Convergence plot of the error in third harmonic amplitude $a_{3}$ at $\omega / \omega_{1}=0.338$ using HBNR and the $k$ - (IGA) and $p$-method (FEM)

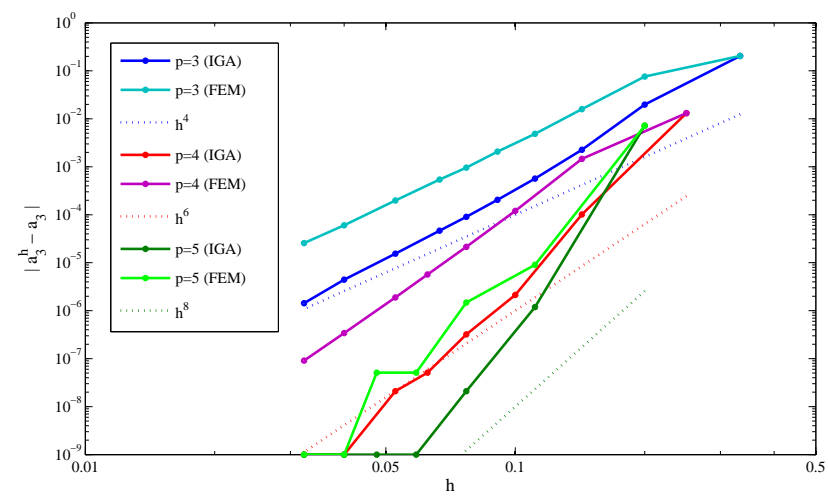

Fig. 22 Convergence plot of the error in third harmonic amplitude $a_{3}$ at $\omega / \omega_{1}=0.338$ using IHB and the $k$ - (IGA) and $p$-method (FEM)

\begin{tabular}{|c|cc|cc|c|}
\hline \multirow{2}{*}{$m$} & \multicolumn{2}{|c|}{ IHB } & \multicolumn{2}{c|}{ HBNR } & $\Delta$ \\
& $a_{3}$ & $\varepsilon$ & $a_{3}$ & $\varepsilon$ & \\
\hline 3 & 0.08756419 & $2.773 \mathrm{E}-01$ & 0.08762534 & $2.774 \mathrm{E}-01$ & $6.115 \mathrm{E}-05$ \\
4 & 0.11645563 & $3.886 \mathrm{E}-02$ & 0.11654205 & $3.892 \mathrm{E}-02$ & $8.642 \mathrm{E}-05$ \\
5 & 0.11649878 & $3.850 \mathrm{E}-02$ & 0.11658537 & $3.856 \mathrm{E}-02$ & $8.659 \mathrm{E}-05$ \\
6 & 0.12128710 & $1.017 \mathrm{E}-03$ & 0.12138500 & $1.018 \mathrm{E}-03$ & $9.790 \mathrm{E}-05$ \\
7 & 0.12116643 & $2.072 \mathrm{E}-05$ & 0.12126404 & $2.078 \mathrm{E}-05$ & $9.761 \mathrm{E}-05$ \\
8 & 0.12116678 & $2.360 \mathrm{E}-05$ & 0.12126439 & $2.367 \mathrm{E}-05$ & $9.761 \mathrm{E}-05$ \\
9 & 0.12116386 & $4.952 \mathrm{E}-07$ & 0.12126145 & $5.773 \mathrm{E}-07$ & $9.759 \mathrm{E}-05$ \\
10 & 0.12116392 & $0.000 \mathrm{E}+00$ & 0.12126152 & $0.000 \mathrm{E}+00$ & $9.760 \mathrm{E}-05$ \\
11 & 0.12116392 & $0.000 \mathrm{E}+00$ & 0.12126152 & $0.000 \mathrm{E}+00$ & $9.760 \mathrm{E}-05$ \\
\hline
\end{tabular}

Table 4 Comparison table of $a_{3}$ at $\omega / \omega_{1}=0.336$ using IHB and HBNR, $p=5, \ell=18$ with varying $m$

\section{Summary and conclusions}

The goal of this paper was the study of isogeometric finite elements within the framework of nonlinear structural vibrations using the harmonic balance method.
As an example for the application of these methods we selected a nonlinear Euler-Bernoulli beam model and validated it by computing the static deformation using isogeometric analysis. The numerical examples showed a higher accuracy of the isogeometric B-Spline $k$-method compared to the $p$-method.

Then both nonlinear vibration analysis methods HBNR and IHB were examined using the $k$-method and $p$-method by comparison with the results of reference work in literature using standard FEM and $p$-FEM. Detailed convergence studies for another computational example showed that vibration analysis with HBNR and IHB using IGA is more accurate for the $k$-method than for the $p$-method, which means that higher smoothness of solution basis functions within the isogeometric approach is very beneficial for numerical results.

We can conclude that the methods, implementations and computational examples introduced and examined in this paper show a promising perspective for the use of isogeometric finite elements within the framework of nonlinear structural vibration analysis. For the analysis of large-scale industrial problems the application of modal reduction techniques seems very useful to lower the number of degrees of freedom in the computation, which increases rapidly using the harmonic balance method. In the future, further studies on other nonlinear structural models like plates, shells or solids and nonlinear materials will be carried out.

Acknowledgements The authors were supported by the 7th Framework Programme of the European Union, project TERRIFIC (FP72011-NMP-ICT-FoF 284981) [40].

\section{References}

1. J.H. Ginsberg. Mechanical and structural vibrations: theory and applications. John Wiley \& Sons, 2001.

2. T.J.R. Hughes. The Finite Element Method: Linear Static and Dynamic Finite Element Analysis. Dover Publications, Mineola, New York, 2000.

3. G.E. Farin. Curves and Surfaces for CAGD: A Practical Guide. Morgan Kaufmann, San Francisco, CA, USA, 2002.

4. L. Piegl and W. Tiller. The NURBS book. Springer-Verlag, London, UK, 1995.

5. D.F. Rogers. An Introduction to NURBS With Historical Perspective. Academic Press, San Diego, CA, USA, 2001.

6. T.J.R. Hughes, J.A. Cottrell, and Y. Bazilevs. Isogeometric analysis: Cad, finite elements, nurbs, exact geometry and mesh refinement. Computer Methods in Applied Mechanics and Engineering, 194(39-41):4135 - 4195, 2005.

7. B. Szabó and I. Babuška. Finite Element Analysis. John Wiley \& Sons, Inc., 1991.

8. Y. Bazilevs, V.M. Calo, T.J.R. Hughes, and Y. Zhang. Isogeometric fluid-structure interaction: theory, algorithms, and computations. Computational Mechanics, 43(1):3-37, 2008.

9. J.A. Cottrell, A. Reali, Y. Bazilevs, and T.J.R. Hughes. Isogeometric analysis of structural vibrations. Computer Methods in Applied Mechanics and Engineering, 195(41-43):5257 - 5296, 2006. 
10. A.-V. Vuong, C. Heinrich, and B. Simeon. Isogat: A 2d tutorial matlab code for isogeometric analysis. Computer Aided Geometric Design, 27:644-655, 2010.

11. M. Ziani, R. Duvigneau, and M. Dörfel. On the role played by nurbs weights in isogeometric structural shape optimization. In International Conference on Inverse Problems, Control and Shape Optimization, Cartagena, Spain, April 2010.

12. M. Aigner, C. Heinrich, B. Jüttler, E. Pilgerstorfer, B. Simeon, and A.-V. Vuong. Swept volume parameterization for isogeometric analysis. In Mathematics of Surfaces XIII, volume 5654 of Lecture Notes in Computer Science, pages 19-44. Springer Berlin / Heidelberg, 2009.

13. Y. Bazilevs, L. Beirão de Veiga, J.A. Cottrell, T.J.R. Hughes, and G. Sangalli. Isogeometric analysis: approximation stability and error estimates for h-refined meshes. Math. Models Methods Appl. Sci., 16(7):1031-1090, 2006.

14. M. Dörfel, B. Jüttler, and B. Simeon. Adaptive isogeometric analysis by local $h$-refinement with t-splines. Comput. Methods Appl. Mech. Engrg., 199(5-8):264-275, 2010.

15. T.J.R. Hughes, A. Reali, and G. Sangalli. Efficient quadrature for nurbs-based isogeometric analysis. Computer Methods in Applied Mechanics and Engineering, 199(5-8):301 - 313, 2010.

16. L. Beirão da Veiga, A. Buffa, J. Rivas, and G. Sangalli. Some estimates for $h-p-k$-refinement in isogeometric analysis. Numer. Math., 118:271-305, 2011.

17. A.-V. Vuong, C. Gianelli, B. Jüttler, and B. Simeon. A hierarchical approach to adaptive local refinement in isogeometric analysis. Computer Methods in Applied Mechanics and Engineering, 200(49-52):3554-3567, 2011

18. J.A. Cottrell, T.J.R. Hughes, and Y. Bazilevs. Isogeometric Analysis: Toward Integration of CAD and FEA. John Wiley \& Sons, 2009.

19. A.A. Ferri. On the equivalence of the incremental harmonic balance method and the harmonic balance-newton raphson method. Journal of Applied Mechanics, 53(2):455-457, 1986.

20. A.H. Nayfeh and B. Balachandran. Applied Nonlinear Dynamics: Analytical Computational, and Experimental Methods. Wiley Series in Nonlinear Science. John Wiley \& Sons, 1995.

21. A.H. Nayfeh and D.T. Mook. Nonlinear Oscillations. Wiley Classics Library. John Wiley \& Sons, 1995.

22. W. Szemplinska-Stupnicka. The Behaviour of Nonlinear Vibrating Systems. Kluwer Academic Publishers, Dordrecht Boston London, 1990.

23. D. Wagg and S. Neild. Nonlinear Vibration with Control: For Flexible and Adaptive Structures. Solid mechanics and its applications. Springer, 2010.

24. K. Worden and G. R. Tomlinson. Nonlinearity in structural dynamics: detection, identification and modelling. Institute of Physics Pub., 2001.

25. R. Lewandowski. Non-linear, steady-state vibration of structures by harmonic balance/finite element method. Computers \& Structures, 44(1-2):287-296, 1992.

26. R. Lewandowski. Computational formulation for periodic vibration of geometrically nonlinear structures, part 1: Theoretical background; part 2: Numerical strategy and examples. International Journal of Solids and Structures, 34(15):1925-1964, 1997.

27. P. Ribeiro and M. Petyt. Non-linear vibration of beams with internal resonance by the hierarchical finite element method. Journal of Sound and Vibration, 224(15):591-624, 1999.

28. P. Ribeiro. Hierarchical finite element analyses of geometrically non-linear vibration of beams and plane frames. Journal of Sound and Vibration, 246(2):225-244, 2001.

29. P. Ribeiro. Non-linear forced vibrations of thin/thick beams and plates by the finite element and shooting methods. Computers and Structures, 82(17-19):1413 - 1423, 2004.

30. Y. K. Cheung, S. H. Chen, and S. L. Lau. Application of the incremental harmonic balance method to cubic non-linearity systems. Journal of Sound and Vibration, 140(2):273 - 286, 1990.
31. S. H. Chen, Y. K. Cheung, and H. X. Xing. Nonlinear vibration of plane structures by finite element and incremental harmonic balance method. Nonlinear Dynamics, 26:87-104, 2001.

32. J.N. Reddy. An Introduction to Nonlinear Finite Elements. Oxford University Press, New York, 2004.

33. D. Gross, W. Hauger, and P. Wriggers. Technische Mechanik 4 - Hydromechanik, Elemente der Höheren Mechanik, Numerische Methoden. Springer-Verlag, Berlin Heidelberg, 7. auflage edition, 2009.

34. N. A. Bobylev, Y. M. Burman, and S. K. Korovin. Approximation procedures in nonlinear oscillation theory. De Gruyter series in nonlinear analysis and applications. W. de Gruyer, 1994.

35. M. Schneider, U. Wever, and Q. Zheng. Parallel harmonic balance. VLSI 93, Proceedings of the IFIP TC10/WG 10.5 International Conference on Very Large Scale Integration, Grenoble, France, 7-10 September, 1993, pages 251-260, 1993.

36. E. L. Allgower and K. Georg. Introduction to Numerical Continuation Methods. Colorado State University, 1990.

37. W. E. Haisler, J. A. Stricklin, and J. E. Key. Displacement incrementation in non-linear structural analysis by the self-correcting method. International Journal for Numerical Methods in Engineering, 11(1):3-10, 1977.

38. T. Belytschko, W.K. Liu, and B. Moran. Nonlinear Finite Elements for Continua and Structures. John Wiley \& Sons, 2000.

39. J.T. Oden. Finite elements of nonlinear continua. Dover Civil and Mechanical Engineering Series. Dover Publications, 2006.

40. TERRIFIC. Towards enhanced integration of design and production in the factory of the future through isogeometric technologies. EU Project FP7, FoF-ICT-2011.7.4. 